\title{
Modeling of Polystyrene under Confinement: Exploring the Limits of Iterative Boltzmann Inversion
}

\author{
Beste Bayramoglu ${ }^{\dagger, \ddagger}$ and Roland Faller ${ }^{\dagger, *}$ \\ ${ }^{\dagger}$ Department of Chemical Engineering \& Materials Science, UC Davis, Davis, California 95616, United States \\ ${ }^{\ddagger}$ Department of Food Engineering, Izmir Institute of Technology, Izmir, Turkey 35430
}

\section{Supporting Information}

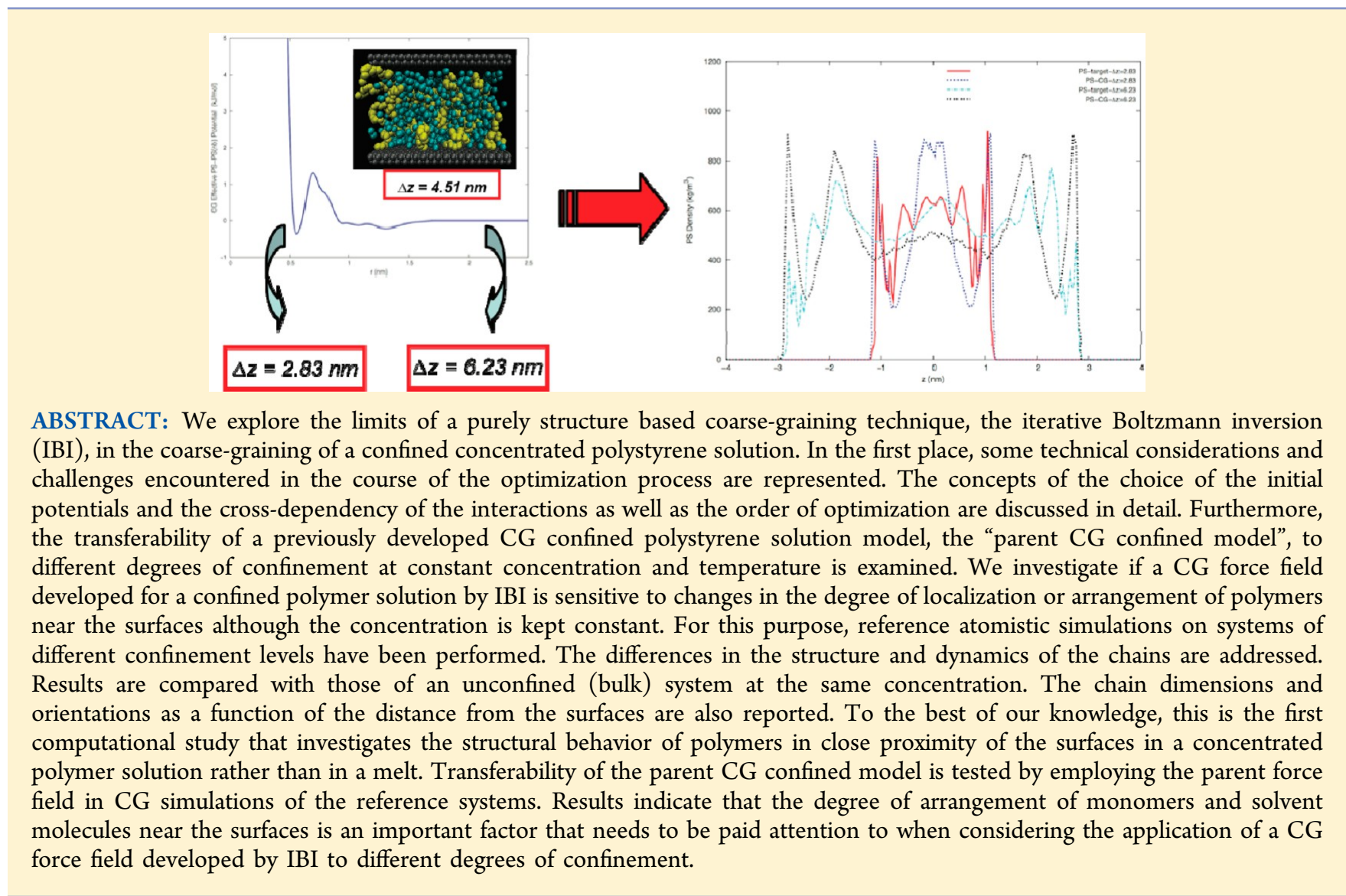

\section{INTRODUCTION}

Polymers are present in every aspect of our lives due to their versatile applications. ${ }^{1}$ An important application of polymers is their use as lubricants or adhesives, ${ }^{2}$ which generates the need to improve our understanding of the structure, thermodynamics, and rheology of polymers near solid surfaces. ${ }^{1}$ Molecular dynamics simulations are complementary to experiments and are a great tool to study structural and dynamic properties of polymers. Modeling polymers, however, is a challenging task due to the enormous variety of length and time scales involved in the description of their structural and dynamic properties. ${ }^{3}$ The use of mesoscale models, which link the length and time scales in higher resolution to those in coarser-level representations, is essential, as a single modeling approach cannot capture all relevant properties. $^{3-6}$ The development of mesoscale models is called "coarse-graining", which can be defined as the derivation of a less detailed model (incorporating fewer degrees of freedom by grouping several atoms into superatoms) from a detailed atomistic model. ${ }^{7}$

If the aim of coarse-graining is to reproduce the structure of the higher resolution model, a number of computational methods are available. ${ }^{8}$ According to Milano et al. the basic idea goes back to Soper, ${ }^{9}$ which was adapted and named iterative Boltzmann inversion (IBI) by Reith et al. ${ }^{10}$ IBI reproduces the structure of the underlying atomistic system by means of an iterative optimization scheme through which the effective

Received: April 23, 2013

Revised: July 31, 2013

Published: September 17, 2013 
potentials between superatoms (CG sites) are obtained. ${ }^{3}$ It is motivated by Henderson's theorem ${ }^{11}$ which states that there is a unique mapping between the radial distribution function $(g(r))$ and the intermolecular potential $(V)$ for simple pairwise additive and spherically symmetric potentials at a given thermodynamic state point: ${ }^{12}$

$$
V=-k_{\mathrm{B}} T \ln (g(r))
$$

eq 1 is actually a potential of mean force and it equals the potential energy only in the limit of infinite dilution. ${ }^{10}$ According to Chan et al. ${ }^{12}$ this is only valid for particles or molecules with a single interaction site rather than molecules with multiple interaction sites (like polymers) since the relation does not account for orientation correlations. However, this does not prohibit its use in the iteration algorithm (see eq 5) for the coarse-graining of polymeric systems with IBI, as the algorithm just serves as a numerical path among many possible ones that yield one effective CG potential by satisfying the condition that the trial CG potential converges when the corresponding conformational distribution matches the reference distribution in the atomistic simulations ${ }^{12}$ and no uniqueness is assumed.

What makes IBI attractive is the use of numerical potentials rather than less flexible analytical potentials in the force field development that also enables the automatization of the whole process minimizing human intervention. ${ }^{4,13}$ However, an important drawback of the method is the strong state-point dependence and limited transferability of the potentials. ${ }^{4,13-16}$ Nevertheless, studies showed that the temperature transferability can be improved through a careful choice of mapping point $(s)^{17}$ and/or inclusion of sufficient chemical detail. ${ }^{18,19}$

Recently, we have developed separate CG models of polystyrene (PS) in various environments (melt, dilute solution, unconfined and confined concentrated solution) using IBI. ${ }^{3}$ The performance of the technique in terms of reproduction of the local and global structures and speed-up in dynamics was studied for each system. Developing mesoscale models for confined polymers is more challenging compared to bulk systems, since one has to account for not only the intermolecular interactions between the chains and surface, but also the surface geometry and chemistry. ${ }^{3,19}$ As a consequence, there are only few reports on coarse-graining of polymers in close contact with surfaces. ${ }^{19-22}$ Moreover, considering the density fluctuations near surfaces in confined systems, ${ }^{1,20,23,24}$ it is even more challenging to develop a CG model by means of a structure based coarse-graining technique such as IBI as the system is locally in different state points. ${ }^{3}$ In the previous publication, ${ }^{3}$ we briefly addressed some of the challenges faced during our implementation of the IBI method on coarsegraining of the confined concentrated PS solution. Here, we go deeper into those challenges as well as some technical considerations in the first part, giving better insight into the limits of IBI technique on coarse-graining of confined heterogeneous polymeric systems. In the later part of the manuscript, the transferability of the developed CG model to different levels of confinement is explored. Considering the density fluctuations near the surfaces, it would be interesting to see how much the model we developed (which is referred as the "parent CG confined model" in the second part) is sensitive to different local states close to the surfaces. For this purpose, we employed the same "parent" force field in CG simulations of two different confined PS solution systems, which have the same number of molecules with different separation distances between the walls. These systems differ in the confinement surface area as the decrease/increase in the volume of the system by more/less confinement is compensated by an increase/decrease of the surface area to keep the concentration constant, which is expected to have an impact on the degree of localization (or arrangement) of polymer chains near the surfaces. In a more general scope, we aim to examine if IBI optimized CG potentials are sensitive to quantitative differences in localization of PS monomers close to the surfaces. Therefore, we explore the limits of iterative Boltzmann inversion technique in developing CG potentials that can be used for a range of confinement levels (at constant concentration and temperature). Besides, the differences in the local and global structure and the dynamics of the chains in different confinement levels are also addressed based on the results of the corresponding reference atomistic simulations. In order to make an appropriate comparison, previous simulation results of an unconfined (bulk) system (of the same concentration $)^{1,3}$ are also presented. To the best of our knowledge, this is the first computational study that reports the structural behavior of polymers in the vicinity of surfaces in a concentrated solution rather than in a melt.

\section{COARSE-GRAINED FORCE FIELD PARAMETERIZATION OF POLYSTYRENE SOLUTION UNDER CONFINEMENT}

The main focus of this part is to get deeper into the technical details and considerations in the course of the coarse-grained (CG) force field parametrization of a confined concentrated polystyrene (PS) solution of which the results were reported in a previous publication. ${ }^{3}$ The challenges faced during the implementation of the iterative Boltzmann inversion (IBI) method are discussed further and conclusions/suggestions on several important points are presented. In IBI, the CG model is developed from the results of a reference atomistic simulation. We have reported the methodological details and results of the atomistic simulations earlier. ${ }^{1,3}$ In short, the reference system is composed of 48 PS chains of 15 monomers with a ratio of meso to racemic dyads of $1: 1$ solvated in 745 toluene molecules confined between opposing graphene surfaces (each composed of two sheets) separated by $4.51 \mathrm{~nm}$ in $z$-direction. The allatom model for PS was taken from earlier studies. ${ }^{6,25}$ All nonbonded interactions between atoms no further than three bonds away within a chain were excluded. Bond lengths were constrained using the LINCS algorithm. ${ }^{26} \mathrm{~A}$ united-atom toluene model, where $\mathrm{CH}$ and $\mathrm{CH}_{3}$ parts are pseudo atoms, was used. ${ }^{27}$ The interaction between graphene walls (fixed in space) and the rest of the system was described by a 6-12 Lennard-Jones potential. ${ }^{28}$ Coulomb interactions were treated by the particle mesh Ewald (PME) method ${ }^{29,30}$ with a cutoff of $1.5 \mathrm{~nm}$, while the cutoff for the van der Waals interactions was $1.7 \mathrm{~nm}$. The temperature was kept at $450 \mathrm{~K}$ by the weakcoupling scheme $\mathrm{e}^{31}$ with time constants of $0.1-0.5$ ps during the initial stages of equilibration, then it was switched to NoseHoover ${ }^{32,33}$ T-coupling with a time constant of 0.5 ps.

Coarse-graining started with the extraction of superatom centers (see section 2.2 for the description) from the output configuration of the reference atomistic simulation: i.e., the final coordinates were used as the initial configurations for the CG simulations. The initial setup of CG walls was described in detail previously. ${ }^{3}$ Numerical potential tables for the CG simulations were generated as follows: All bond length distributions were fitted to Gaussians; dihedral distributions 
were smoothed by cubic splines. Bending angle distributions, radial distribution functions (RDFs) and corrected potentials were smoothed by cubic splines when needed. To conserve total energy, the minima of the corrected bonded potentials were shifted to zero, and the corrected nonbonded potentials beyond the cutoff $(r \geq 1.7 \mathrm{~nm})$ were set to zero by shifting the whole potential preserving the shape. ${ }^{3}$

The technical details in the CG simulations are the same as in atomistic simulations. All CG simulations were carried out using GROMACS (version 4.0.7) in-36 $^{36 T}$ ensembles under orthorhombic periodic boundary conditions. The time steps used in the atomistic and CG simulations were 2 and 5 fs, respectively. Production runs lasted for 20 and 40 ns for atomistic and CG simulations, respectively.

2.1. Coarse-Graining Methodology. In iterative Boltzmann inversion (IBI), atomistic simulations are used as calibration in terms of the structure. ${ }^{14}$ The method has been described in detail elsewhere $;^{10,37,38}$ so we limit ourselves to a brief explanation of the basics. Coarse-graining starts with choosing an appropriate mapping scheme in which a number of atoms (typically 10 to 20 ) are grouped into a superatom (or CG site). One assumes that the total potential energy of the CG system can be separated into bonded and nonbonded interaction energies so that they can be optimized separately. It is further assumed that the conformational distributions (bond, angle, and dihedral angle distributions) are not correlated. Note that this is an approximation since individual distributions are dependent on the full set of potentials through higher-order correlations in dense systems. ${ }^{10}$ However, experience shows that one can largely avoid the correlation of at least bond and bending/dihedral angle distributions with an appropriate choice of mapping scheme/point. ${ }^{4,15}$ The initial potentials are commonly obtained by taking the Boltzmann inverse of the target probability distributions of the atomistic simulation:

$$
V^{C G}(\xi)=-k_{\mathrm{B}} T \ln \left(P^{\text {target }}(\xi)\right)
$$

Here, $\mathrm{P}^{\text {target }}(\xi)$ is the Jacobian transformed target probability distribution of the corresponding degrees of freedom (bond, angle, dihedral distributions or pair correlation functions $g(r)$ ), $k_{\mathrm{B}}$ is the Boltzmann constant and $T$ is the temperature at which the atomistic simulations were carried out.

The distributions calculated at the end of the first trial CG simulation (0th iteration) will deviate from the targets due to packing effects. This requires the modification of the effective interaction potentials, which is done by adding a correction term to the previous trial potential $\mathrm{V}_{\mathrm{i}}^{\mathrm{CG}}(\xi)$ :

$$
V_{i+1}^{C G}(\xi)=V_{i}^{C G}(\xi)+a_{i} k_{\mathrm{B}} T \ln \left[\frac{P_{i}^{C G}(\xi)}{P^{\text {target }}(\xi)}\right]
$$

In eq 3 , the correction term includes a convergence control constant, $a_{\mathrm{i}}$, which normally ranges from 0.05 to 1 depending on the deviation between the calculated distribution, $P_{\mathrm{i}}^{\mathrm{CG}}(\xi)$, and the target, $P^{\text {target }}(\xi)$. The effective potential is iterated until the target distribution is reproduced to a sufficient degree. The degree of convergence of the effective potentials is measured by a merit function:

$$
f_{\text {merit }}=\frac{\int\left[P_{i}^{C G}(\xi)-P^{\text {target }}(\xi)\right]^{2} \mathrm{~d} \xi}{\int\left[P^{\text {target }}(\xi)\right]^{2} \mathrm{~d} \xi}
$$

In this study, iterations were continued until $f_{\text {merit }}<0.003$. Finally, a linear pressure correction term is usually added to the long-range part of the optimized CG nonbonded interaction potentials in case the resulting pressure is different from the atomistic system. ${ }^{10}$ The correction term is of the form

$$
\Delta V(r)=A\left(1-\frac{r}{r_{c}}\right), \quad A \sim-0.1 k_{\mathrm{B}} T
$$

where $r_{\mathrm{c}}$ is the van der Waals cutoff. This step is included only if one aims also to reproduce the thermodynamics of the system as pressure correction might cause some deterioration in the conformational distributions. In such a case, the whole process turns into a cycle in which reoptimizations are performed until both the structure and pressure are reproduced. In this work, no pressure correction was performed considering the amount of deviation from the target pressure and the fact that the effect of pressure correction is seen mostly in the region beyond $r>1$ $\mathrm{nm}$, which is not very important for the structural fit. ${ }^{10}$

2.2. Initial Considerations. The mapping scheme for polystyrene was directly taken from an earlier publication. ${ }^{14} \mathrm{~A}$ single type of superatom, which represents a PS monomer, is used. The mapping point (superatom center) is the carbon atom the phenyl ring is attached to. The scheme simplifies the potential derivation process by minimizing the number of iterations due to use of a single type of superatom and a welldefined bond length between superatoms that comes with our choice of the mapping point. The solvent is toluene where the mapping is straightforward: one superatom represents the whole molecule with its center on its center of mass. ${ }^{3}$

We represent the surfaces by perfectly crystalline walls rather than continuous perfectly smooth surfaces as the latter is known to increase the diffusive dynamics of the polymers near the walls. ${ }^{21}$ Each CG wall bead possesses the mass of a PS monomer (104.06 amu). Each surface is composed of two sheets of 1368 beads arranged on a hexagonal lattice. The closest distance between beads is $0.254 \mathrm{~nm}$ (bond length of a PS monomer). The surfaces are $l_{x}=8.36 \mathrm{~nm} \times l_{y}=6.86 \mathrm{~nm}$, with a separation distance of $5.095 \mathrm{~nm}$ in the $z$-direction, and fixed in space. ${ }^{3}$ Although this kind of representation of the CG surfaces simplifies the procedure by decreasing the total number of iterations, it introduces some unknowns to the process. As the CG wall beads are arbitrary, there is no information about the interactions between the beads and the rest of the system. Furthermore, since there is no mapping within the walls, there are no target conformational distributions between the wall beads and PS monomers/TOL molecules to build the effective potentials. The only structural information we have from atomistic simulations is the density profiles of PS monomers and TOL molecules. This suggests that the aim should be to reproduce the density profiles as closely as possible together with the target conformational distributions in the reference system. Regarding the interactions associated with the surfaces, their interaction with each other was excluded while the interactions with the rest of the system were described by 12-6 Lennard-Jones type potentials. The choices of appropriate parameters are explained below.

2.3. Choice of Initial Potentials. The iteration process needs a reasonable initial guess potential to get started and the use of Boltzmann inverse of the target conformational distribution is common and usually sufficient to start the optimizations.

Chan et al. ${ }^{12}$ studied the effect of use of different initial potentials on the final effective potentials and concluded that, although they produce similar RDFs, different initial guess 


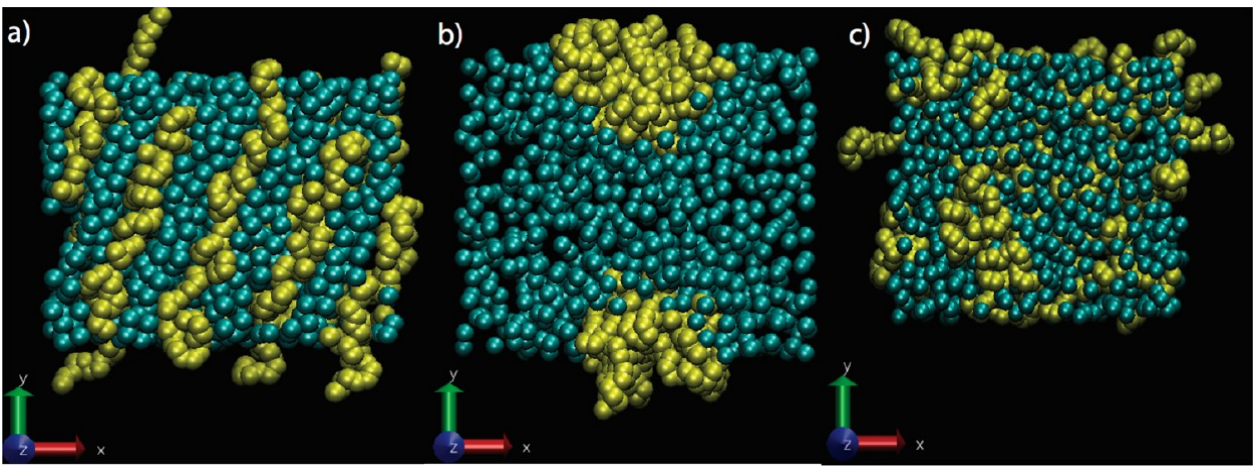

Figure 1. Visualization of the system (PS superatoms, yellow; TOL superatoms, blue) (a) at the end of the 1st trial (0th iteration) with the use of Boltzmann inverse functions of the target conformational distributions as the initial guess potentials. The polymers are in a semiglassy state. (The interactions between the walls and PS superatoms are described by previously optimized CG PS-PS effective nonbonded potentials in the CG PS melt system while the interactions between the walls and TOL superatoms are described by previously optimized CG PS-TOL effective potentials in the CG dilute PS solution system (see inset of Figure 3). (b) PS micelles solvated by TOL molecules after a few iterations on PS-PS and TOLTOL potentials. The iterations were started on a semiglassy state like in part a (except that there are no confining walls in this system). The system turned into a solution only when PS-TOL pair potential was treated or when the dihedral potential was fixed together with manually changing the TOL-TOL potential before all the other pair potentials (not shown). (c) at the end of the 1st trial (0th iteration) using the optimized CG effective potentials of the unconfined concentrated solution system as the initial potentials (see section 2.3.2). The interactions between the walls and PS monomers/TOL molecules are described by LJ potentials and shown in the inset of Figure 3. The walls are located in $z$-direction confining the system (except part b) and not shown for clarity.).
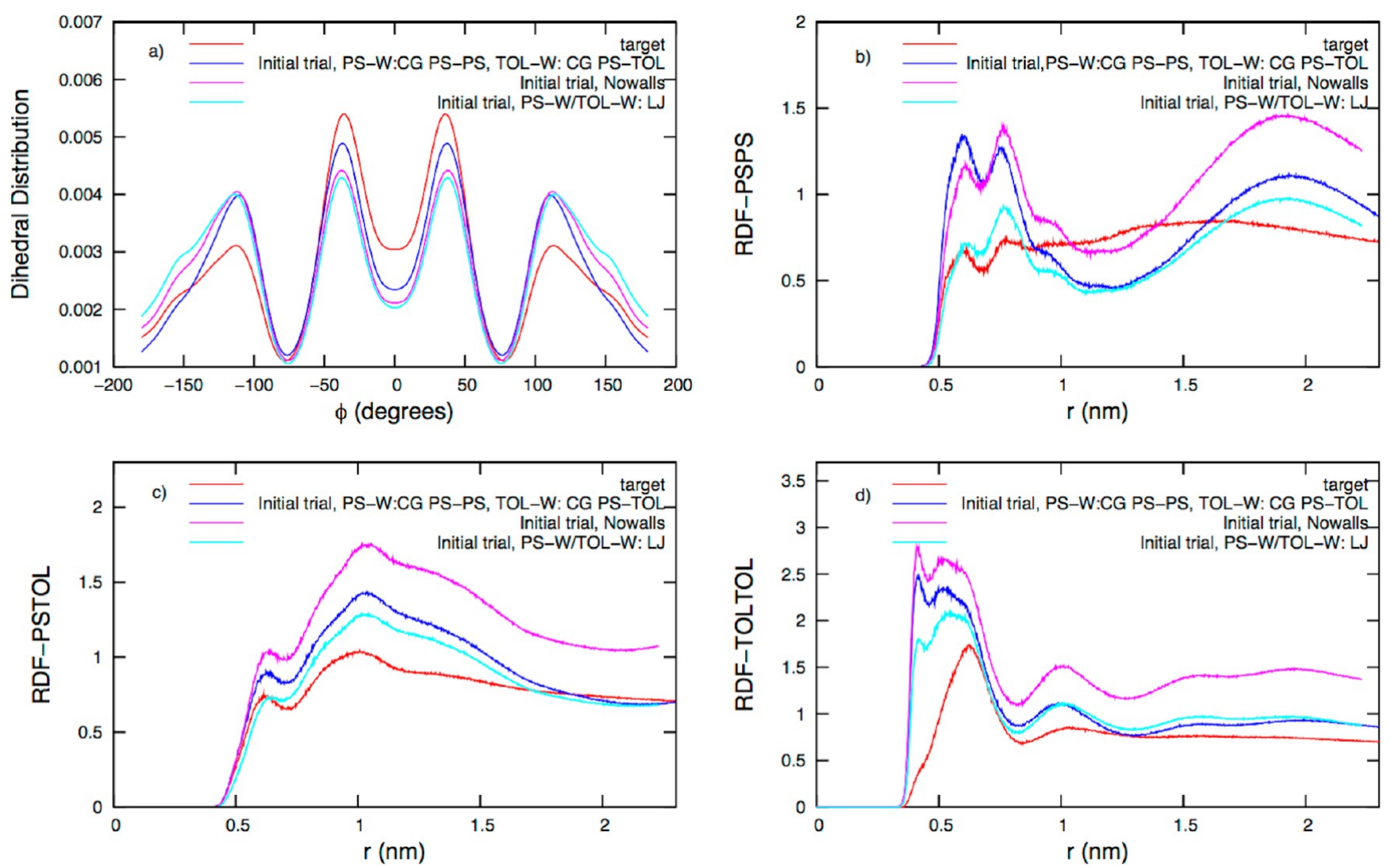

Figure 2. Dihedral distribution (a) and radial distribution functions (b, PS-PS; c, PS-TOL; d, TOL-TOL) between PS monomers and TOL molecules at the end of 1st trial (0th iteration) with the use of Boltzmann inverse functions of the target conformational distributions as the initial guess potentials. The plot includes the results for three alternative initial trials: (i) with walls that interact with PS superatoms through previously optimized CG effective nonbonded PS-PS potential of a melt ${ }^{3}$ (denoted as "CG PS-PS") and with TOL superatoms through previously optimized CG effective PS-TOL potential of a dilute solution ${ }^{3}$ (denoted as "CG PS-TOL"), (ii) without walls, and (iii) with walls that interact with the rest of the system through $12-6 \mathrm{LJ}$ potentials. See text for explanation.

potentials could reproduce similar effective pair potentials at low temperatures but not at high temperatures, which suggests that the CG potentials obtained by IBI are not unique.
In our implementation of the method, the iterations were started with the Boltzmann inverses of the target conformational distributions. However, the system turned into a 
semiglassy state in the first trial (0th iteration) where some polymers are ordered. In the following subsections, we discuss the details of this transition, demonstrating that using the Boltzmann inverses of the target distributions as the initial potentials is not always a convenient approach (for confined systems). Afterward, we present the results obtained by use of an alternative set of initial potentials.

2.3.1. Boltzmann Inverse Functions of the Target Distributions. We started with the Boltzmann-inverse functions of the target distributions between CG superatoms extracted from the atomistic confined concentrated PS solution system. The initial guess potentials between the wall beads and the rest of the system were chosen arbitrarily. The optimized CG nonbonded PS-PS potential of the CG melt system was directly used for the interactions between the walls and PS superatoms, while the optimized CG PS-TOL potential of the CG dilute solution system was directly used for the interactions between the walls and TOL superatoms (see inset of Figure 3). We should note that the choice of this set of potentials is an arbitrary initial guess for the interactions between the walls and the polymer solution, as one cannot expect a total agreement due to the imbalance in entropic components (wall beads fixed in space vs free chains/molecules).

At the end of the initial trial (0th iteration), it was observed that some polymers crystallized in the solution and the system entered a semiglassy state as shown in Figure 1a. This behavior was reflected in some conformational distributions. It was observed that bond and bending angle distributions were not significantly off from the reference distributions (not shown), but the dihedral distribution and the RDFs were, as shown in Figure 2 .

The transformation of the system into a semiglassy state is explained by the imbalance between the initial potentials (Figure 3). Overall, the attractive interactions between PS monomers and TOL molecules cannot compete with those between TOL molecules resulting in toluene becoming a worse solvent. However, the transformation of the polymers into a

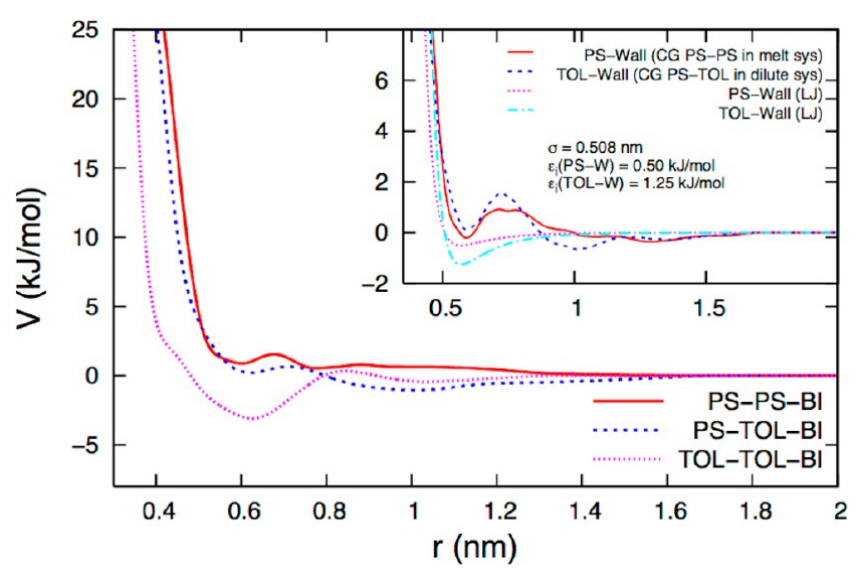

Figure 3. Boltzmann inverse functions (BI) of the target radial distribution functions in the atomistic confined concentrated solution system, which were used as the initial potentials for the CG simulations (0th iteration). Inset: Different sets of initial PS-wall and TOL-wall interactions used in the 0th iteration. Same axes labels are valid for the subplot. "CG PS-PS in melt sys" stands for the optimized CG effective nonbonded PS-PS potentials of the melt system where "CG PS-TOL in dilute sys" stands for the optimized CG effective PS-TOL potentials of the dilute solution system (see text). semiglassy state was mostly aided with the dihedral angle potential (not shown) and purely repulsive nonbonded interactions between the monomers. The dihedral distribution in Figure 2a indicates that polymers are more extended than in the reference system, which is probably the reason for the formation of a system in semiglassy state composed of mostly stretched chains.

In order to see the effect of walls and their interactions with PS and TOL superatoms on this phase transition (falling out of solution), two more independent initial trial simulations were carried out: one excluding the walls and one with walls interacting with the rest through 12-6 LJ type potentials (the same ones that were used with the new set of initial potentials as described in section 2.3.2) (see inset of Figure 3). In both of these trials, Boltzmann inverse functions of the target distributions were used as the initial potentials for the interactions between PS and PS/TOL superatoms. However, polymers were still in glassy state. The resulting dihedral distribution and the RDFs are also shown in Figure 2. It is clear from the distributions that the presence of walls, which interact with the system through attractive forces (even though they are weak), softens the conformational distributions between PS and PS/TOL superatoms. Furthermore, the stronger these interactions are, the softer the distributions are. Nevertheless, they were not effective enough as the system was still in semiglassy state, which points that the situation seems to be related mostly to the imbalance between the initial guess potentials used for PS-PS, PS-TOL, and TOL-TOL interactions.

At this point, one should manually alter the potentials instead of pursuing the iteration process in order to save time. This is because the initial dihedral and nonbonded distributions are far from the target distributions as a consequence of the phase transformation. One needs to turn it back into a solution first. Furthermore, the speed of convergence is considerably affected by the order of optimization, ${ }^{10}$ which is not a priori known in heterogeneous systems, but often the order can be inferred after a few initial trials. ${ }^{39}$ The system could be turned back into a solution only when we worked on the PS-TOL interactions. All attempts to optimize TOL-TOL and PS-PS potentials only led to micelles surrounded by TOL molecules (Figure $1 \mathrm{~b}$ ). Alternatively, the system was a solution when the dihedral potential was fixed together with manually changing the TOLTOL interactions before all the other nonbonded potentials.

As there is no strong theoretical basis for using the Boltzmann inverse functions of the target distributions in the reference system as the initial guess potentials, ${ }^{12}$ we completely discarded them and used previously optimized CG potentials for the unconfined solution system at the same concentration, ${ }^{3}$ which saved a lot of time as the optimization was completed in only 18 iterations.

\subsubsection{Optimized CG Effective Potentials of the Unconfined} Concentrated Solution System. The iteration procedure was restarted using the new set of initial potentials (see Figure 4); i.e., previously optimized (and pressure corrected) CG effective potentials of the unconfined PS solution system of the same concentration. $^{3}$ Initial interactions between the CG walls and the rest of the system were described by 12-6 type LJ potentials. Alternative sets of LJ parameters were tried and the one, which gave the best density profiles was chosen (Figure 5). The system did not phase separate at the end of the initial trial (Figure 1c), because the initial potentials are not substantially different from the final potentials as seen from Figure 4. The 

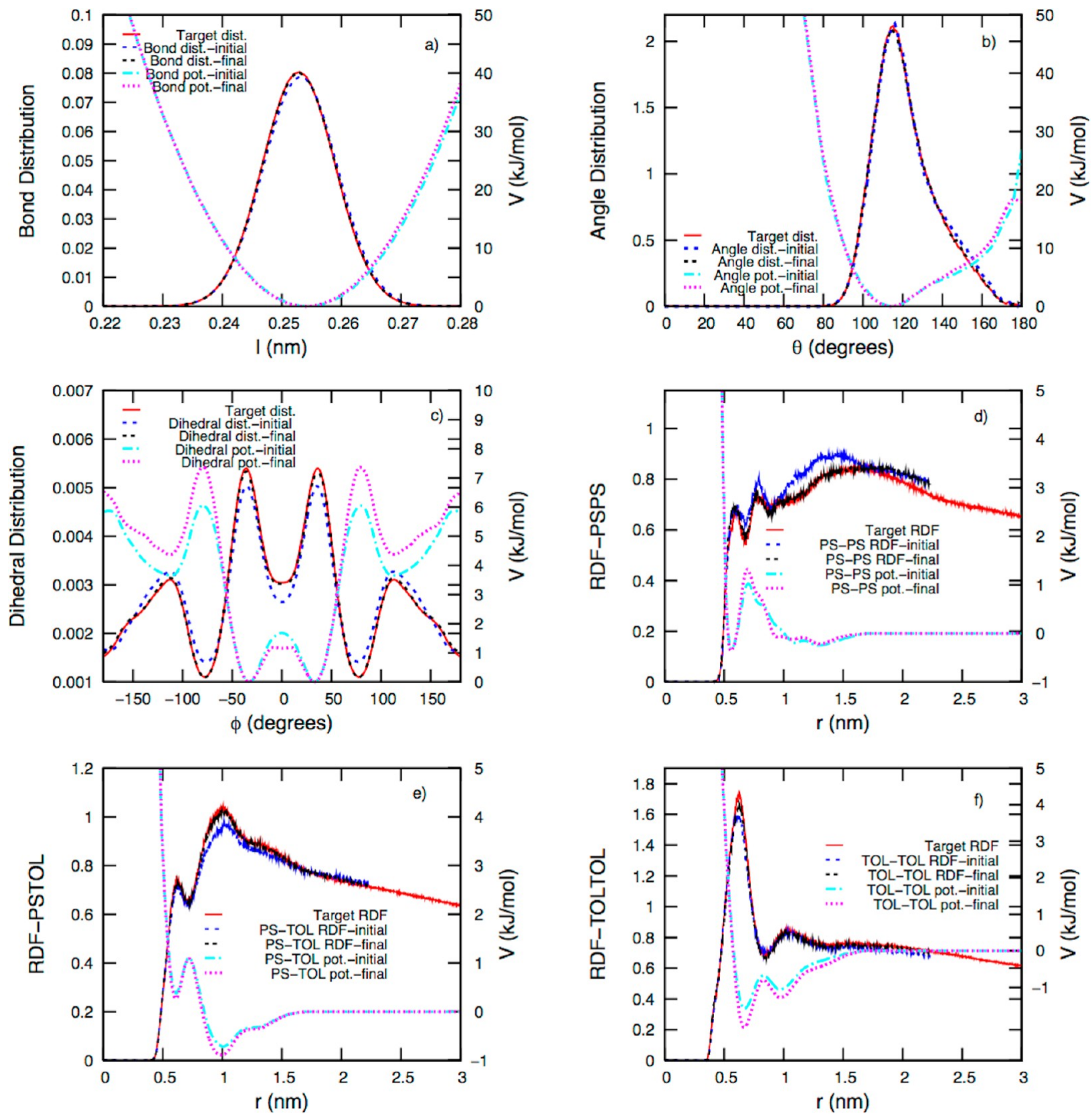

Figure 4. Initial and final [(a) bond, (b) angle, (c) dihedral, (d) nonbonded PS-PS, (e) PS-TOL, and (f) TOL-TOL] distributions/potentials of the CG confined concentrated solution system. Initial potentials are the final optimized and pressure corrected CG potentials of the unconfined concentrated system of the same concentration and the initial distributions are the resulting structures with the use of these potentials. Final potentials are the optimized (not pressure corrected) potentials at the end of the iteration process and the final distributions are the resulting structures (reproductions of the target structures) obtained by the use of these potentials.

iterations were continued until both the target conformational distributions and the density profiles (not shown) were reproduced sufficiently. However, as mentioned before, the overall process was a cycle of reoptimizations due to the correlations especially between nonbonded interactions. During the process, the interactions between the surfaces and the solution were manually modified a few times. Final potentials are shown in Figure 5. We refer the reader to the previous publication $^{3}$ for detailed discussions on the differences in final PS-wall and TOL-wall interactions as well as the resulting density profiles.

We finalized the coarse-graining process without correcting the pressure as the deviation $(\sim-15$ bar $)$ from the overall target pressure $(-16 \mathrm{bar}$, due to the negative lateral pressure caused by anisotropic P-coupling ${ }^{1}$ ) is tolerable compared to the effort to go through postoptimization. Because of the inherent complexity within the system, laborious work was needed further to fix both the structure and pressure. As a matter of fact, previous experience showed that there is no significant difference in the overall structure and static properties of the polymers in a blend with or without pressure correction. 3,40

In summary, the results show that, for the coarse-graining of confined polymer systems with IBI, using the Boltzmann inverses of the target distributions may not be convenient to start the optimizations, as it might lead to unintended consequences such as phase transformations. This is because 


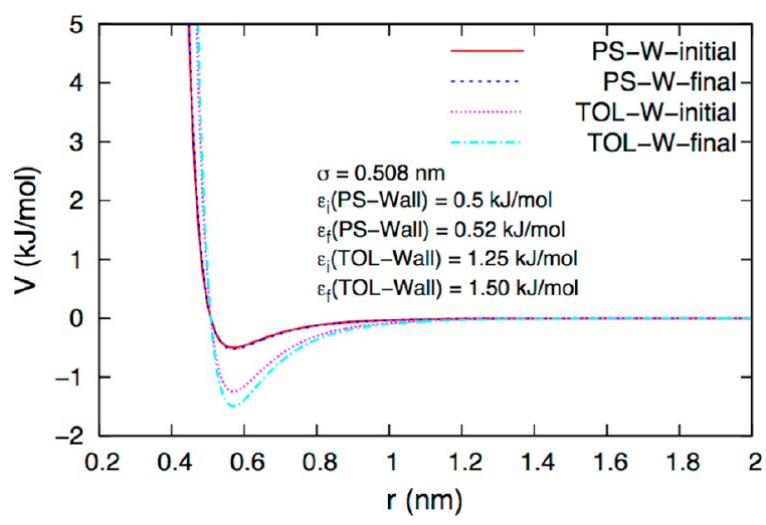

Figure 5. Initial guess potentials and final LJ potentials between the CG walls and PS/TOL superatoms.

the potentials of mean force (Boltzmann inverse functions) accounts neither for the higher order nonpairwise correlations nor the PS orientation correlations (induced by the connectivity of the PS monomers plus the presence of walls) in the system. On the other hand, first coarse-graining the system without confinement and then using these optimized potentials as the initial potentials to start the iterations on the confined system might be a good alternative.

2.4. Cross-Dependency of the Interactions and the Order of Optimization. The main assumptions in IBI are that the effective potentials are independent of each other ${ }^{10,41}$ and that the nonbonded interactions are pairwise. ${ }^{42}$ However, this is not strictly true for dense and/or heterogeneous systems. It is known that individual conformational distributions depend on all potentials in dense systems. ${ }^{10}$ The situation is even more complicated for heterogeneous systems composed of molecules with multiple interaction sites (like polymers). In that case, the orientation correlations must also be accounted for. ${ }^{12}$ Therefore, during the iteration process, corrections on one type of potential (and its corresponding distribution) usually alter the other(s) making reoptimizations unavoidable. For practical purposes, one should start with potentials that are least affected by changes to all other ones; i.e., the stiffest ones. This is why the order of optimization typically follows stretching, bending angle, nonbonded and dihedral angle. ${ }^{10}$

In coarse-graining of polymeric systems, the choice of the mapping scheme is an important factor, which influences the
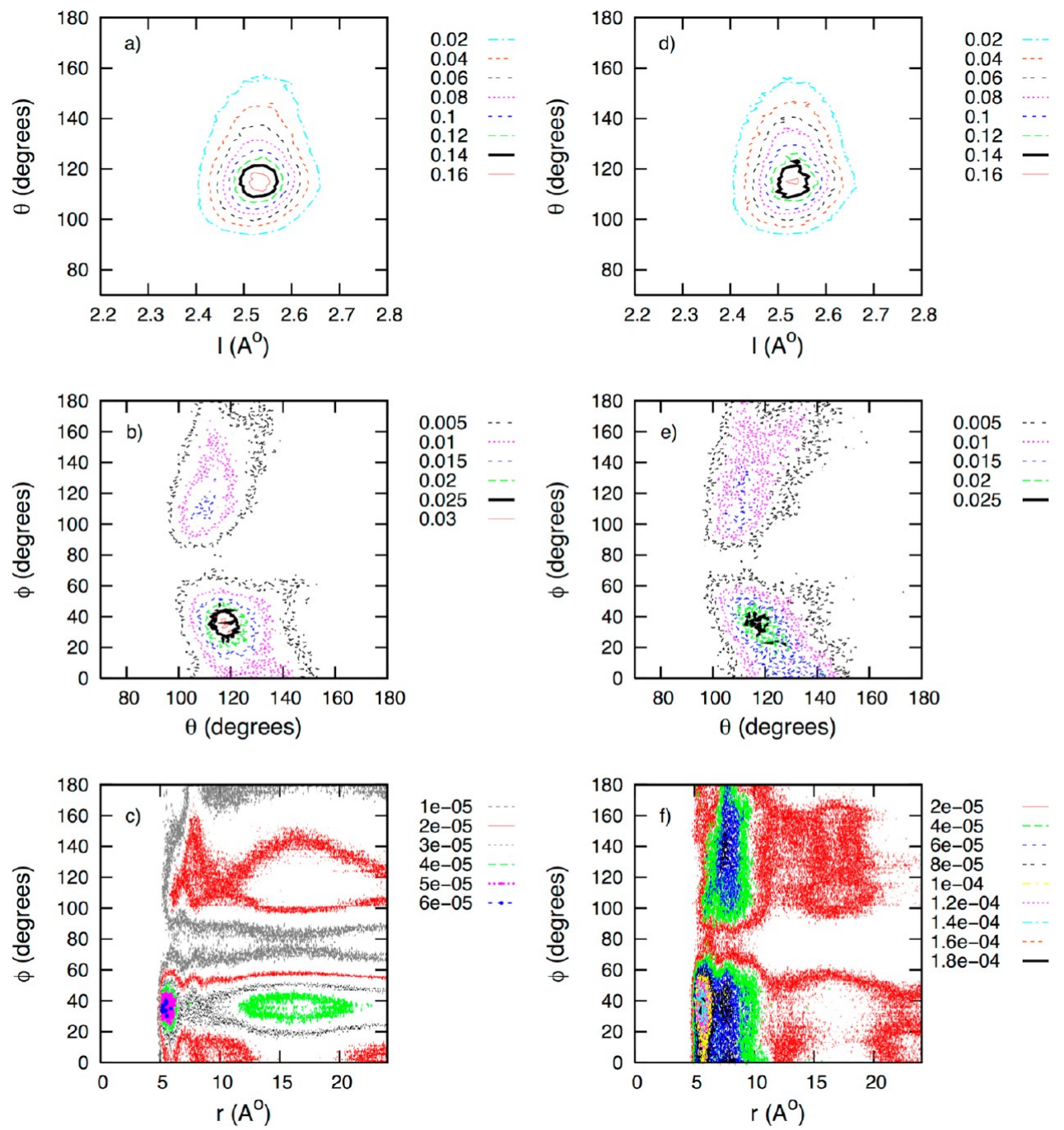

Figure 6. Contour plots of joint probability distributions between bond length $(l)$ and bending angle $(\theta)$, bending angle $(\theta)$ and dihedral angle $(\phi)$, and nonbonded PS-PS RDF $(r)$ and dihedral angle $(\phi)$ of PS monomers/superatoms for all PS chains in the system $(a-c)$ and for only the chains close to the surfaces $(\mathrm{d}-\mathrm{f})$. Calculations are performed on the last $2 \mathrm{~ns}$ of the atomistic simulations. 
correlation of individual distributions. A good mapping scheme provides at least uncorrelated bond length and bending angle distributions. An appropriate way to check the dependency of different types of distributions is to calculate the 2-D joint probability distributions between them. Figure 6 gives insight into the nature of the correlations in the current system through the joint bond length-bending angle, bending angledihedral angle and nonbonded PS-PS RDF-dihedral angle probability distributions between PS superatoms (monomers). As it is interesting to see if/how these cross-correlations differ for surface segments, the same calculations were performed solely on the chains near the surfaces (which were determined as described in section 3.1.2). We should note that the results reported in Figure 6 are from atomistic simulations. In order to check if the correlations are reproduced sufficiently by our CG model, the same calculations were performed on the CG simulations. A comparison between the atomistic and CG results is available in the Supporting Information. Briefly, few slight differences were found in the joint bending angle-dihedral angle distributions; i.e., some regions that were not sampled in the atomistic simulations were sampled in the CG simulations. This is clearly an artifact of the decoupled bonded distributions assumption. An additional 1-4 repulsive interaction can be introduced between PS superatoms to overcome this problem. ${ }^{41}$ However, since the peaks (i.e., most favorable conformations) were reproduced correctly and the deviations from the atomistic results were mostly in rarely sampled regions, we concluded that the current CG force field sufficiently reproduces the cross-correlations without further modification.

Turning back to Figure 6a, it is seen that the bond length and angle distributions are not completely independent. However, the bond length is very well-defined and its 1-D bond length distribution (Figure 4a) is very close to Gaussian. Because of the stiffness of the bond stretching potential, once the bond length distribution was fixed by optimizing the stretching potential in the first iteration, it never needed to be reoptimized. This shows that the bond length distribution is very weakly correlated (if at all) with the other distributions in the current mapping scheme. Given that, it is not surprising to observe no significant differences in the same type of crosscorrelation for only the chains near the surfaces (Figure 6d).

In contrast, the mapping scheme does not decorrelate bending and dihedral angles (Figure 6b,e); i.e. in the course of the iteration process, modifications in one potential altered the distribution of the other. Because of the stiffer nature of the bending potential, angle distribution was optimized within a few iterations. For all the chains in the system, we find that larger bending angles $\left(\theta>140^{\circ}\right)$ only appear with smaller dihedral angles $\left(\theta<65^{\circ}\right)$ (Figure $\left.6 \mathrm{~b}\right)$. However, the peak value in the angle distribution $\left(\theta \sim 115^{\circ}\right)$, which is mainly correlated with the smaller peak $\left(\sim \pm 35^{\circ}\right)$ in the dihedral distribution, shows also a weaker correlation with the dihedral peak at $\sim \pm 110^{\circ}$. In Figure 6e, the joint bending angle-dihedral angle probability distribution only for the chains near the surfaces is given. It is clear that the ranges sampled in both $\theta$ and $\phi$ are very similar as those in Figure $6 \mathrm{~b}$. The most prominent difference is that the correlations are less localized for surface chains; i.e. each bending angle in the range $110^{\circ}-140^{\circ}$ is correlated with a larger range of dihedrals. Moreover, it is observed that cis conformations are more strongly correlated with bending angles $120-140^{\circ}$ in surface chains while the correlation between $\theta \approx 115^{\circ}$ and $\phi \approx \pm 35^{\circ}$ is weaker than the overall. Certainly, all of these differences are also seen in the individual bending angle and dihedral distributions in Figure 10 (see section 3.1.1).

We also find a close relationship between the first peak of the PS-PS RDF and the smaller peak value of the dihedral distribution $\left( \pm 35^{\circ}\right)$. Whenever the dihedral potential at $\sim \pm 35^{\circ}$ was corrected; the first peak of the PS-PS RDF was fixed as well. The correlation between the two for all the chains and for surface segments only is clearly seen in Figure 6, parts $\mathrm{c}$ and $\mathrm{f}$. Note that the strength of this correlation in surface chains is 2 times more than the average for all chains in the system.

Earlier, we mentioned that the order of optimization in IBI is typically the stretching bond, bending angle, nonbonded, and dihedral angle potentials based on the strength of the interactions. However, the order of the last two might change for the first few trials unless the initially calculated dihedral distribution is close to the target. During our first attempts of the implementation of the method using Boltzmann inverse functions as the initial guess potentials (section 2.3.1), we observed that it was impossible to improve the first peak of the PS-PS RDF without correcting the dihedral distribution first. Since the initial dihedral distribution was significantly off (Figure 2a), its negative effect on the first peak of PS-PS RDF was substantial. On the contrary, by using an alternative set of initial potentials (section 2.3.2), there was no need for treatment of the dihedral potential first since all initial distributions were already close to the targets (Figure 4). Therefore, in case any initial dihedral distribution(s) is/are far from the target(s), we suggest that it/they should be iterated before the treatment of nonbonded self-interactions, as this might be crucial for the optimization of the local region in the RDFs.

Figure 6c, also shows that the dihedrals in the range $\pm \sim 30^{\circ}$ to $\pm \sim 50^{\circ}$ are strongly correlated with the structures at larger distances $(\sim 1.25 \mathrm{~nm}<r<\sim 2.1 \mathrm{~nm})$ while this correlation is missing for surface segments (Figure $6 \mathrm{f}$ ). For larger dihedral angles $\left(>\mid \sim 90^{\circ}\right)$, the correlations are weak as they are much less sampled than the small ones; Figure $6 \mathrm{c}, \mathrm{f}$ also show that the weak shoulder at $\phi>\mid \sim 150^{\circ}$ | (see Figure $4 \mathrm{c}$ ), which appears to be a clear peak for surface segments (Figure 10e), is mostly correlated with the second peak of the PS-PS RDF. Another clear distinction between surface chains and the average is that the dihedral angle ranges with which the first two peaks of PSPS RDF are associated are larger for surface segments resulting in less localized correlations similar to Figure 6e. This is a consequence of the increase in the probability of cis conformations as well as dihedrals larger than $130^{\circ}$ in the chains near the surfaces (Figure 10e). Finally, some additional cross-correlations $\left(12 \AA<r<20 \AA\right.$ and $100^{\circ}<\phi<140^{\circ}$, etc. $)$ are observed for surface segments.

The nature of the correlations between nonbonded distributions is hard to define explicitly. The correlations are complicated due to nonpairwise contributions to all the nonbonded interactions as well as the effect of orientation correlations induced by the connectivity of the PS monomers plus the presence of walls. As expected, nonbonded PS pair potentials are the most demanding with regards to the need for reoptimization. Nevertheless, due to a good set of initial guess potentials, the optimization process was completed in 18 iterations. Finally, we discuss the strength of the effect of PS in the system with an example. During the initial iterations, we observed that it was not possible to improve the TOL-TOL RDF without improving the PS-TOL interactions first, 
although the initial TOL-TOL RDF was close to the target RDF (Figure 4f). This is because, effectively, polystyrene rules the system due to the connectivity of the monomers. Local chain packing and orientation correlations have a considerable effect.

\section{SIMULATIONS OF CONFINED POLYSTYRENE SOLUTION UNDER DIFFERENT DEGREES OF CONFINEMENTS}

We now study the effect of confinement level on the structure and dynamics of PS chains first atomistically and then coarsegrained. We compare the results with those in the bulk (unconfined) system of the same concentration. Then we test the transferability of our previously developed CG confined concentrated solution model ${ }^{3}$ to different confinement levels keeping the overall concentration of the system and the temperature constant. We call this model/system the "parent CG confined system/model", and the reference atomistic confined concentrated solution system/model the "parent reference confined system/model” from now on (see Table 1).

Table 1. Details of the Atomistic and CG Confined Systems ${ }^{a}$

$\begin{array}{lllll} & H / R_{\mathrm{g}_{\mathrm{b}}} & \begin{array}{c}\Delta z \\ (\mathrm{~nm})\end{array} & \begin{array}{c}\Delta z_{\text {effective }} \\ (\mathrm{nm})\end{array} & \begin{array}{c}\text { simulation box } x y \\ (\mathrm{~nm} \times \mathrm{nm})\end{array} \\ \begin{array}{l}\text { atomistic } \\ \text { simulations }\end{array} & 3.91 & 2.83 & 2.425 & 10.806 \times 9.359 \\ & 6.23^{b} & 4.51^{b} & 4.1^{b} & 8.351 \times 7.231^{b} \\ & 8.62 & 6.23 & 5.825 & 7.122 \times 5.956 \\ \text { CG simulations } & & 3.208 & 2.45 & 11 \times 9.145 \\ & & 5.095^{c} & 4.32^{c} & 8.36 \times 6.8^{c} \\ & & 6.531 & 5.75 & 7.04 \times 6.097\end{array}$

${ }^{a} \Delta z$ is the separation distance between the walls and $\Delta z_{\text {effective }}$ is the effective distance in $z$-direction that the solution occupies, which is determined based on the density profiles. Each system is composed of 48 PS chains (of 15 monomers) and 745 toluene molecules. ${ }^{b}$ System details for the "parent reference confined system". ${ }^{c}$ System details for the "parent CG confined system".

3.1. Atomistic Simulations. Effect of Confinement Level. The methodological details and results of the atomistic simulations of the parent reference confined system and the bulk system were reported earlier. ${ }^{1}$ The reference system, with 48 PS chains of 15 monomers and 745 TOL molecules, is confined between opposing graphene surfaces separated by $4.51 \mathrm{~nm}$ in $z$-direction. This corresponds to an $H / R_{\mathrm{g}_{\mathrm{b}}}$ ratio of 6.23 , where $H$ is the distance between the surfaces $(\Delta z)$ and $R_{\mathrm{g}_{\mathrm{b}}}$ is the average radius of gyration of a PS chain in the bulk solution (composed of 48 PS chains and 778 TOL molecules). ${ }^{1}$ Keeping the overall effective concentration of the system the same as the reference $\left(963.3 \mathrm{~kg} / \mathrm{m}^{3}\right)$, two additional confinement levels are studied: a smaller confinement with $H / R_{\mathrm{g}_{\mathrm{b}}}=$ $3.91(\Delta z=2.83 \mathrm{~nm})$ and a larger confinement with $H / R_{\mathrm{g}_{\mathrm{b}}}=$ $8.62(\Delta z=6.23 \mathrm{~nm})$.

The equilibration runs for the smaller confinement system started with the final configuration of the equilibrated parent system except that the graphene surfaces were larger in size $\left(10.806 \times 9.359 \mathrm{~nm}^{2}\right.$, see Table 1) to compensate for the decrease in height. The initial distance between the walls (4.6 $\mathrm{nm}$ ) was decreased to $2.83 \mathrm{~nm}$ in 59 steps. At each step the system was relaxed for 5 ps after the distance between the surfaces was reduced by $0.03 \mathrm{~nm}$. Preparation of the initial configuration of the larger confinement system was more cumbersome. We started again with the final configuration of the parent system rotated $90^{\circ}$ about the $z$-, and $x$-axes, each. This was performed in order to achieve the desired system size in $x y$-plane $\left(7.122 \times 5.956 \mathrm{~nm}^{2}\right.$, see Table 1$)$ faster. Once the simulation box $x$ and $y$ dimensions were adjusted by gradual alterations of mainly the $y$-box dimension followed by relaxation $\mathrm{MD}$ runs with short time steps $(\mathrm{d} t=0.1 \mathrm{fs})$, the walls were added to the system in $z$-direction with an initial separation distance of $11.12 \mathrm{~nm}$. The reason for such a large initial separation was to prevent any possible overlaps between solution and wall atoms. Then, the distance between the walls was reduced to $6.23 \mathrm{~nm}$ in 166 steps with relaxation runs for 5 ps between each reduction of $0.03 \mathrm{~nm}$. In all of these simulations, the simulation box dimension in the $z$-direction was $50 \mathrm{~nm}$, which was big enough to avoid interactions between periodic images. ${ }^{1,28}$ All the equilibration runs were performed at constant temperature $(450 \mathrm{~K})$ controlled by the weak-coupling scheme introduced by Berendsen ${ }^{31}$ with a time constant of $0.1 \mathrm{ps}$, which was later switched to Nose-Hoover T-coupling ${ }^{32,33}\left(\tau_{\mathrm{t}}=0.5 \mathrm{ps}\right)$ for the production runs. Simulations lasted until the density profiles/end-to-end distance distributions of PS monomers were not significantly changing during the last few $5 \mathrm{~ns}$ increments of the production run. Analyses were made on the last 2 ns of the whole trajectory for both of the systems. All other technical details are the same as described in section 2 .

3.1.1. Local Structure. Differences in the local arrangement of PS monomers in different confinement levels were characterized through bond/angle/dihedral distributions, radial distribution functions (RDFs) between monomer centers (excluding end monomers) and density profiles. Additionally, the answer to the question "Is the effect of confinement, thus the surface arrangement of polymers significant for the chains near the surfaces in terms of bonded conformational distributions?" was searched for. This was achieved by performing the calculations only for those chains, which were the nearest to the surfaces during the last $2 \mathrm{~ns}$ of the corresponding trajectories as described in section 3.1.2.

As local structure is linked with density profiles, we start our discussions on them. Figure 7 shows PS and TOL density profiles for all confinement levels. Only the largest confinement system $(\Delta z=6.23)$ differs from the other systems in terms of PS and TOL surface densities suggesting to categorize systems in terms of average surface concentrations. PS average surface concentrations are close to each other in systems $\Delta z=2.83$ and $\Delta z=4.51\left(0.174 \mathrm{mg} / \mathrm{m}^{2}\right.$ and $0.168 \mathrm{mg} / \mathrm{m}^{2}$, respectively) while it is much smaller $\left(0.09 \mathrm{mg} / \mathrm{m}^{2}\right)$ in $\Delta z=6.23$. Similarly, TOL surface concentrations are close to each other in smaller confinements whereas there is a pronounced adsorption of toluene molecules ( $\sim 3.5$ times of PS) on the walls in $\Delta z=$ 6.23. This can be explained by the order parameter measurements $\left(S_{z}\right)$ of the end-to-end vectors (of PS chains), which gives information about the relative orientation of the chains with respect to the surface normal:

$$
S_{z}=\frac{3}{2}\left\langle\cos ^{2} \Theta\right\rangle-\frac{1}{2}
$$

Here, $\Theta$ is the angle between the end-to-end vector and the surface normal. $S_{z}$ varies between -0.5 and 1 . Values of $-0.5,0$, and 1 mean perpendicular, isotropic, and parallel orientation of end-to-end vectors with respect to the surface normal, respectively. The results in Figure 8 indicate that the chains 

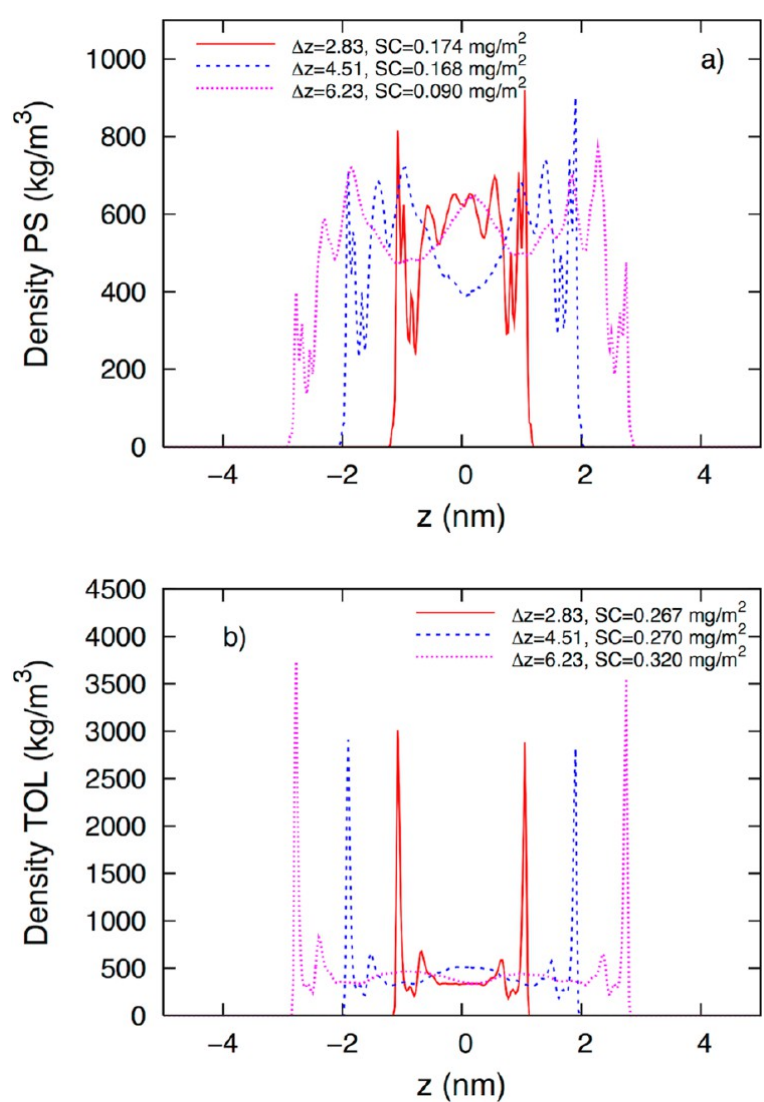

Figure 7. (a) PS and (b) TOL density profiles along $z$-direction for different confinement levels. Corresponding average surface concentrations (SC) in $\mathrm{mg} / \mathrm{m}^{2}$ are also given in the labels.

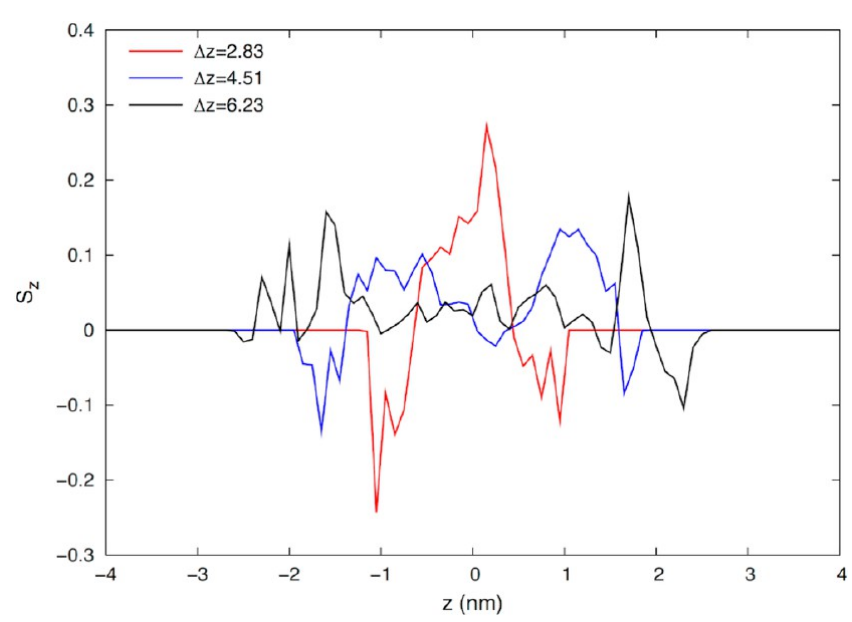

Figure 8. Order parameters $\left(S_{z}\right)$ of end-to-end vectors with respect to the surface normal for different degrees of confinement.

in close proximity with the surfaces are aligned only weakly parallel to the surfaces, as the order parameters are much smaller in magnitude than -0.5 . The degree of parallel alignment in the vicinity of the walls decreases with increasing separation between the walls and almost disappears for the largest confinement system $(\Delta z=6.23)$. It is observed that only a small portion of the chains are aligned weakly parallel to the walls (at $2 \mathrm{~nm}<z<2.5 \mathrm{~nm}$ ), while the rest are aligned weakly normal with respect to the walls (at $-2.5 \mathrm{~nm}>z>-1$ and $1.5 \mathrm{~nm}<z<2 \mathrm{~nm}$ of $\Delta z=6.23$ ). This is the reason behind the conspicuous localization of toluenes near the surfaces in the largest confinement system. As PS chains align less parallel to the surfaces, the space left on the surfaces is preferably filled with TOL molecules, which are smaller and more mobile. Different alignment of polymers near the surfaces in $\Delta z=6.23$ results from the tendency to increase their entropy. Recall that the concentration and the interaction between the walls and PS monomers/TOL molecules are equal in all systems. However, confining surface area is the smallest in $\Delta z=6.23$ system, while the separation distance between the walls is the largest (see Table 1), which provides more freedom to the monomers to arrange/orient themselves in as many ways as possible when compared to the smaller confinement systems. This is also demonstrated in Figure 8; i.e., the chains in the middle of the $\Delta z=6.23$ system show bulklike isotropic orientations not feeling the confinement. Note that random orientations decrease with confinement and finally disappear. The chains in the center of the smallest confinement system show weakly normal alignment with respect to the walls.

End monomer density profiles also support the order parameter results, which suggest that the weak degree of parallel alignment of PS chains near the surfaces increases with the degree of confinement. In Figure 9, the normalized end monomer densities (by multiplication with the ratio of 15 total
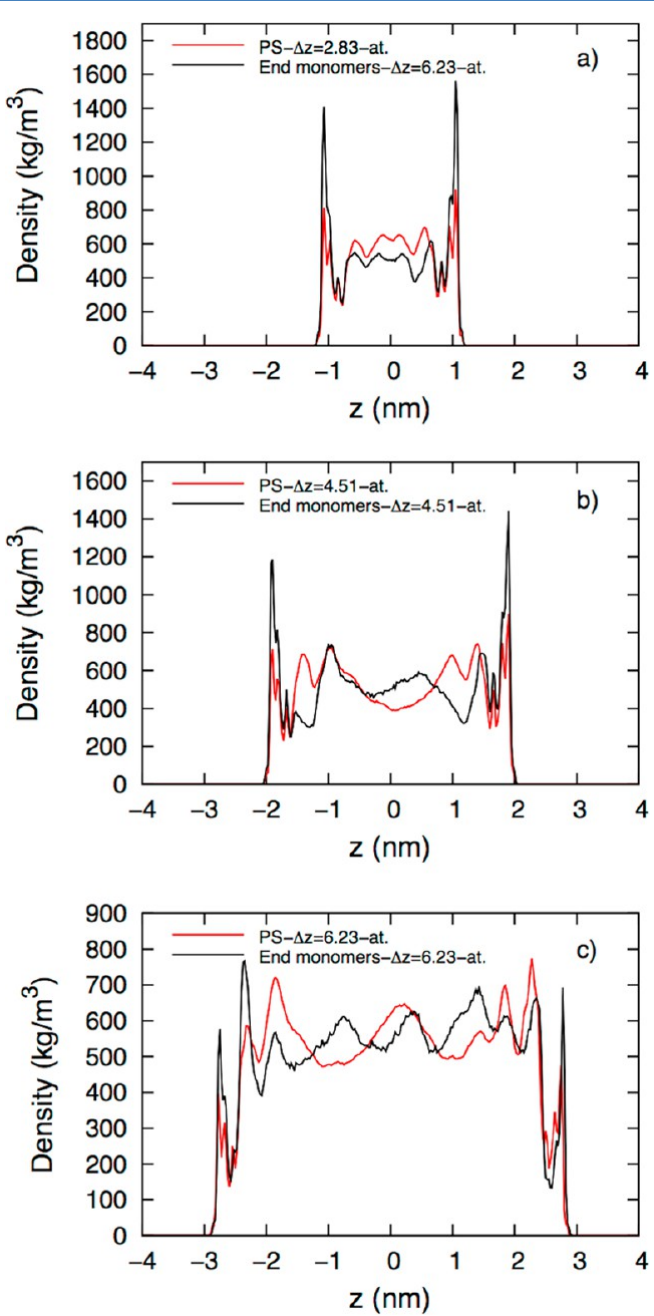

Figure 9. Comparison of PS density profile with normalized end monomer density profile for different degrees of confinement. End monomer profiles are scaled by 7.5 . 

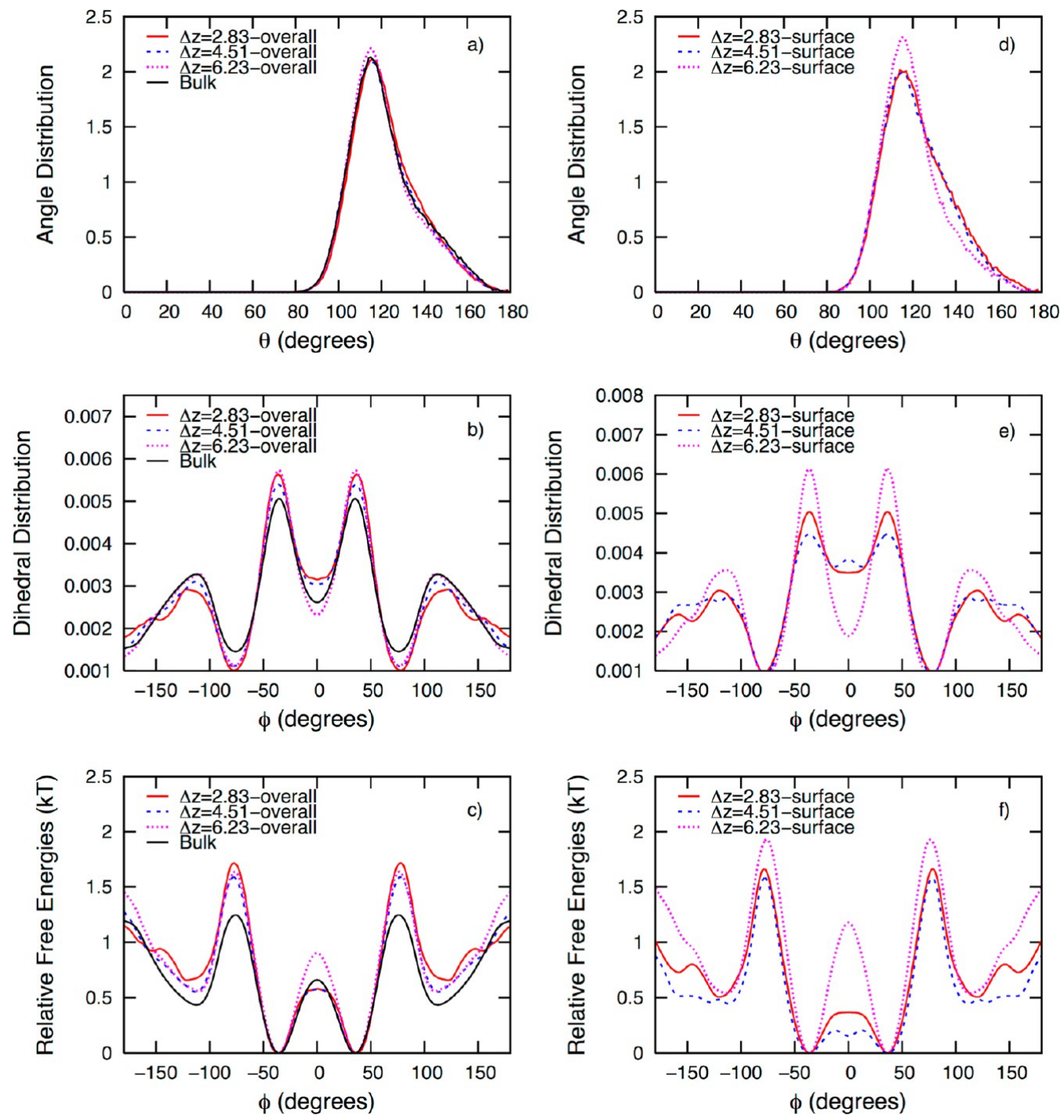

Figure 10. Bonded conformational probability distributions between PS monomers for different confinement levels and corresponding relative free energies in kT for all PS chains in the system $(a-c)$ and for chains near the surfaces only $(d-f)$. The analyses were made on the last 2 ns of the corresponding trajectories.

monomers $/ 2$ end monomers) are compared with the whole PS densities. It is clear that the end monomers migrate closer to the surfaces. End monomer adsorption increases with degree of confinement for two reasons: first, the surface area gets larger as the separation distance between the walls decreases allowing more chains, therefore end monomers, to migrate; second, due to the geometrical restrictions in $z$-direction, chains near the walls adopt relatively more flattened (parallel) conformations compared to larger confinement systems, again allowing more end monomers to migrate closer to the surfaces. ${ }^{19}$ As the chains show more flattened conformations near the walls, fewer chains from the rest of the solution will be able to reach the surfaces resulting in reduced total amount of adsorption. This is why a distinct difference in PS surface adsorption was not observed between $\Delta z=2.83$ and $\Delta z=4.51$ systems, although the confining surface areas were quite different.

Next, we address the bonded conformational distributions. Bond distance between monomer centers was found to be $0.254 \mathrm{~nm}$ and represented by a Gaussian distribution in all systems including the bulk (Figure 4a). Figure 10a shows the overall bending angle distributions (all chains) for the three confinement levels together with the bulk case. It is observed that the distributions are not significantly different from the bulk, at least for larger confinements. Observation of the overall dihedral distributions (Figure 10b) indicates an increase in both compact and more elongated states with confinement. Analysis of static properties (end-to-end distance $\left(R_{\mathrm{ee}}\right)$, parallel and normal components of square of radius of gyration and the ratio $R_{\mathrm{ee}}{ }^{2} / R_{\mathrm{g}}^{2}$ ) of the polymer chains, which characterizes their conformations/shapes, gives a better idea. The chains have relatively more elongated conformations in the smallest confinement system. Comparison of the dihedral distributions in the bulk and confined systems indicates that the chains exhibit compact states less in the bulk than under confinement (probabilities of $\pm 35^{\circ}$ and $0^{\circ}$ are smaller without confinement), which is also indicated by the $R_{\mathrm{ee}}$ calculations. The relative free energies in Figure 10c show that the dihedral interactions are the softest in the bulk as the barriers are less pronounced. Note that almost all barriers are on the order of thermal energy and, therefore, accessible.

Comparison of the bonded distributions for the surface segments with the overall and within different degrees of 
confinement shows clear differences indicating that the surface localization/arrangement of the polymers has a significant effect. In Figure 10d, it is seen that the bending angle distribution of the surface chains in $\Delta z=6.23$ is significantly different from those in smaller confinements. There is an increase in the intensity of the peak angle, $\sim 115^{\circ}$, and a consequent decrease in the probabilities of larger angles $(\theta>$ $130^{\circ}$ ), which can be explained again by the order parameter results. As the surface chains show almost no parallel alignment in $\Delta z=6.23$, the driving force for adopting larger bending angles is diminished. Note that the angle distributions are almost the same in $\Delta z=2.83$ and $\Delta z=4.51$ due to similar PS surface concentrations and alignment behavior near the surfaces.

Inspection of Figure 10e reveals that dihedral distributions are significantly different for surface chains compared to the overall for all the confinement levels studied. Smaller confinement systems $(\Delta z=2.83$ and $\Delta z=4.51)$ show similar behaviors in terms of the change in dihedrals (again due to their similar weakly parallel alignment on the surfaces); i.e., an increase in the probabilities of cis conformations and dihedral angles larger in magnitude than $\sim \pm 150^{\circ}$ is observed accompanied by a decrease in the intensities of $\sim \pm 35^{\circ}$. In contrast, when there is almost no parallel alignment near the surfaces as in $\Delta z=6.23$, it seems like there is no driving force for the segments to adopt additional larger dihedrals. We observe an increase in the probabilities of only the two peak values $\left( \pm 35^{\circ}\right.$ and $\left.\pm 110^{\circ}\right)$ together with a significant decrease in cis conformations. The effect of arrangement of the chains near surfaces is also observed in the corresponding relative free energies (Figure $7 \mathrm{f}$ ). One can easily notice how different they are in systems $\Delta z=4.51$ and $\Delta z=6.23$ for surface chains while they are alike for all the chains (Figure 7c).

Figure 11a shows the local packing of PS monomers through radial distribution functions between nonbonded monomers (excluding up to the third nearest bonded neighbors). All structures of the bulk are reproduced at the same positions in all confined systems. The probability of finding the fourth nearest neighbor to a monomer in a chain plus the first nearest neighbor from other chains (1st peaks) is the same regardless of the degree of confinement. The probability of finding farther monomers seems to decrease as $\Delta z$ increases. In all confined systems, intensities are smaller than in the bulk. This is due to the finite size effect introduced by the presence of walls, i.e. the monomers near the walls in the confined systems inevitably will have fewer neighbors (from other chains) at intermediate and larger distances than the ones at the center of the system. ${ }^{1}$ This is also the reason for negative slopes in the RDFs. The finite size effect increases with the degree of confinement and is the largest in the smallest confinement system $(\Delta z=2.83)$, as expected. Finally, the PS-PS RDF in the largest confinement system $(\Delta z=6.23)$ is close to the bulk at intermediate distances $(\sim 1 \mathrm{~nm} \leq r \leq \sim 1.5 \mathrm{~nm})$. This indicates that most chains do not feel the effect of confinement as suggested by the order parameters of end-to-end vectors (Figure 8). The chains in the center of the system are randomly oriented like in an unconfined system.

Radial distribution functions between monomers and solvent molecules characterize the differences in solvation shells around PS monomers (Figure 11b), while TOL-TOL RDFs monitor the local packing of toluenes around themselves (Figure 11c). All solvation shells are reproduced at the same locations in each system including the bulk, as one would expect TOL molecules
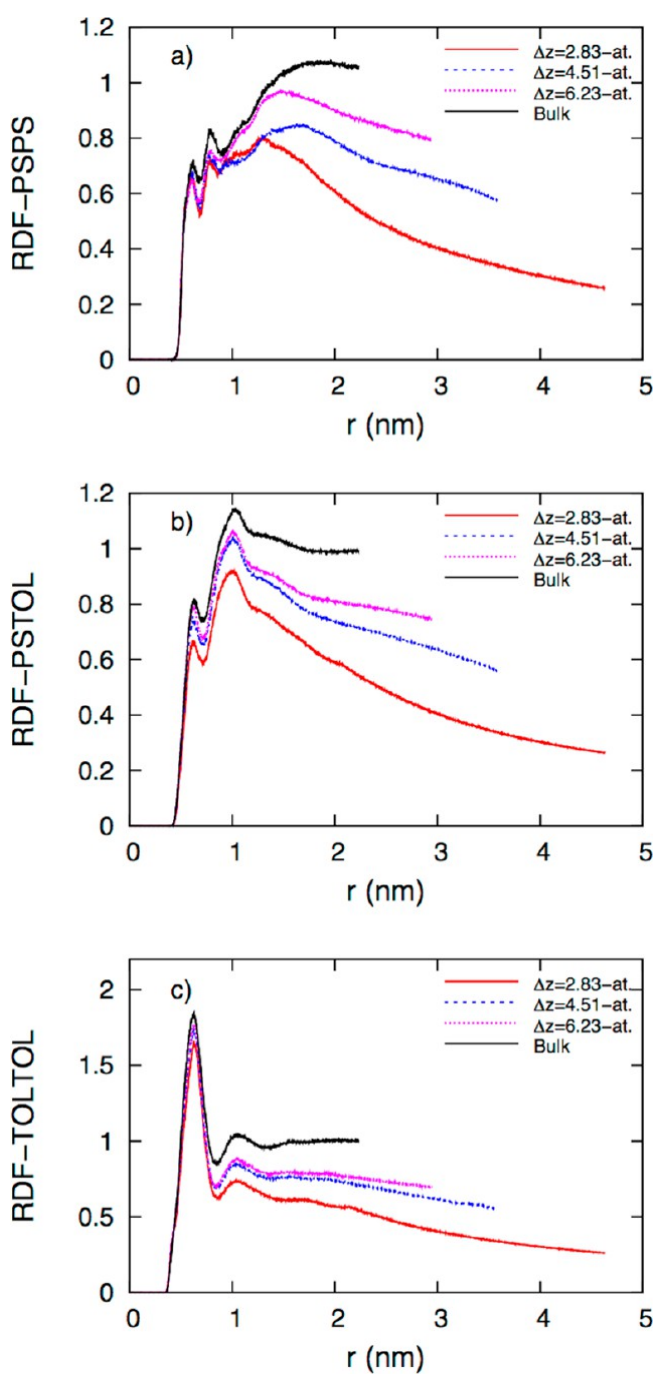

Figure 11. Radial distribution functions between (a) PS monomers (RDF PS-PS), (b) PS monomers and TOL molecules (RDF PSTOL), and (c) TOL molecules (RDF TOL-TOL) for different confinement levels.

to approach PS monomers at the same distances. Note that the same finite-size effects are observed in these RDFs as well; i.e., lower intensities and sharper negative slopes in the tails with increasing confinement.

3.1.2. Static Properties. Static properties of PS chains were investigated by means of end-to-end distances $\left(\mathrm{R}_{\mathrm{ee}}\right)$, radii of gyration $\left(R_{\mathrm{g}}\right)$ and persistence lengths $\left(l_{\mathrm{p}}\right)$ for each system (Table 2). The data for the parent atomistic confined system and the unconfined system were reported earlier. ${ }^{1}$ The results show that $R_{\mathrm{g}}$ is largest in the bulk, while $R_{\mathrm{ee}}$ is largest in the smallest confinement system $(\Delta z=2.83)$. The ratio $R_{\mathrm{ee}}{ }^{2} / R_{\mathrm{g}}{ }^{2}$ provides an estimate for the conformation of a chain; a ratio of 6 represents a random walk, a ratio smaller than 6 indicates a more compact conformation, while a ratio bigger than 6 indicates a more elongated conformation. ${ }^{1,43}$ Therefore, our chains are compact in all systems; they are most elongated under strongest confinement. Elongation increases weakly with degree of confinement. In order to take a closer look to the dimensions of the chains relative to the surfaces, the square of the parallel $\left(\left(R_{\mathrm{g} x}{ }^{2}+R_{\mathrm{g} y}{ }^{2}\right) / 2\right)$ and normal components $\left(R_{\mathrm{g} z}{ }^{2}\right)$ of the radius of gyration were calculated (Table 2$)$. The results indicate that the average chain dimensions parallel to the 
Table 2. Static Properties of PS Chains under Different Confinement Levels and in the Unconfined (Bulk) System

$\begin{array}{lcccccc}\text { system } & R_{\mathrm{g}}(\mathrm{nm}) & R_{\mathrm{ee}}(\mathrm{nm}) & R_{\mathrm{ee}}{ }^{2} / R_{\mathrm{g}}{ }^{2} & \left(R_{\mathrm{g} x}{ }^{2}+R_{\mathrm{g} y}{ }^{2}\right) / 2\left(\mathrm{~nm}^{2}\right) & R_{\mathrm{g} z}{ }^{2}\left(\mathrm{~nm}^{2}\right) & l_{\mathrm{p}}{ }^{a}(\mathrm{bonds}) \\ \Delta z=2.83 & 0.717 \pm 1.1 \mathrm{e}-4 & 1.531 \pm 8.6 \mathrm{e}-4 & 4.57 \pm 4.5 \mathrm{e}-3 & 0.337 \pm 8.8 \mathrm{e}-5 & 0.339 \pm 2.4 \mathrm{e}-4 \\ \Delta z=4.51 & 0.712 \pm 1.0 \mathrm{e}-2 & 1.46 \pm 1.2 \mathrm{e}-3 & 4.22 \pm 6.1 \mathrm{e}-3 & 0.338 \pm 1.1 \mathrm{e}-4 & 0.324 \pm 1.8 \mathrm{e}-4 \\ \Delta z=6.23 & 0.712 \pm 4.0 \mathrm{e}-5 & 1.456 \pm 4.0 \mathrm{e}-4 & 4.18 \pm 2.0 \mathrm{e}-3 & 0.335 \pm 4.9 \mathrm{e}-5 & 0.333 \pm 8.4 \mathrm{e}-5 & 1.33 \pm 2.1 \mathrm{e}-4 \\ \text { bulk } & 0.723 \pm 1.7 \mathrm{e}-4 & 1.477 \pm 1.5 \mathrm{e}-3 & 4.16 \pm 6.8 \mathrm{e}-3 & 0.344 \pm 2.5 \mathrm{e}-4 & 0.347 \pm 3.6 \mathrm{e}-4\end{array}$

${ }^{a}$ Persistence lengths were calculated excluding the end monomers. The bond length between the backbone carbons in a PS chain is $0.153 \mathrm{~nm}$. Standard errors of all the data are stated as well.
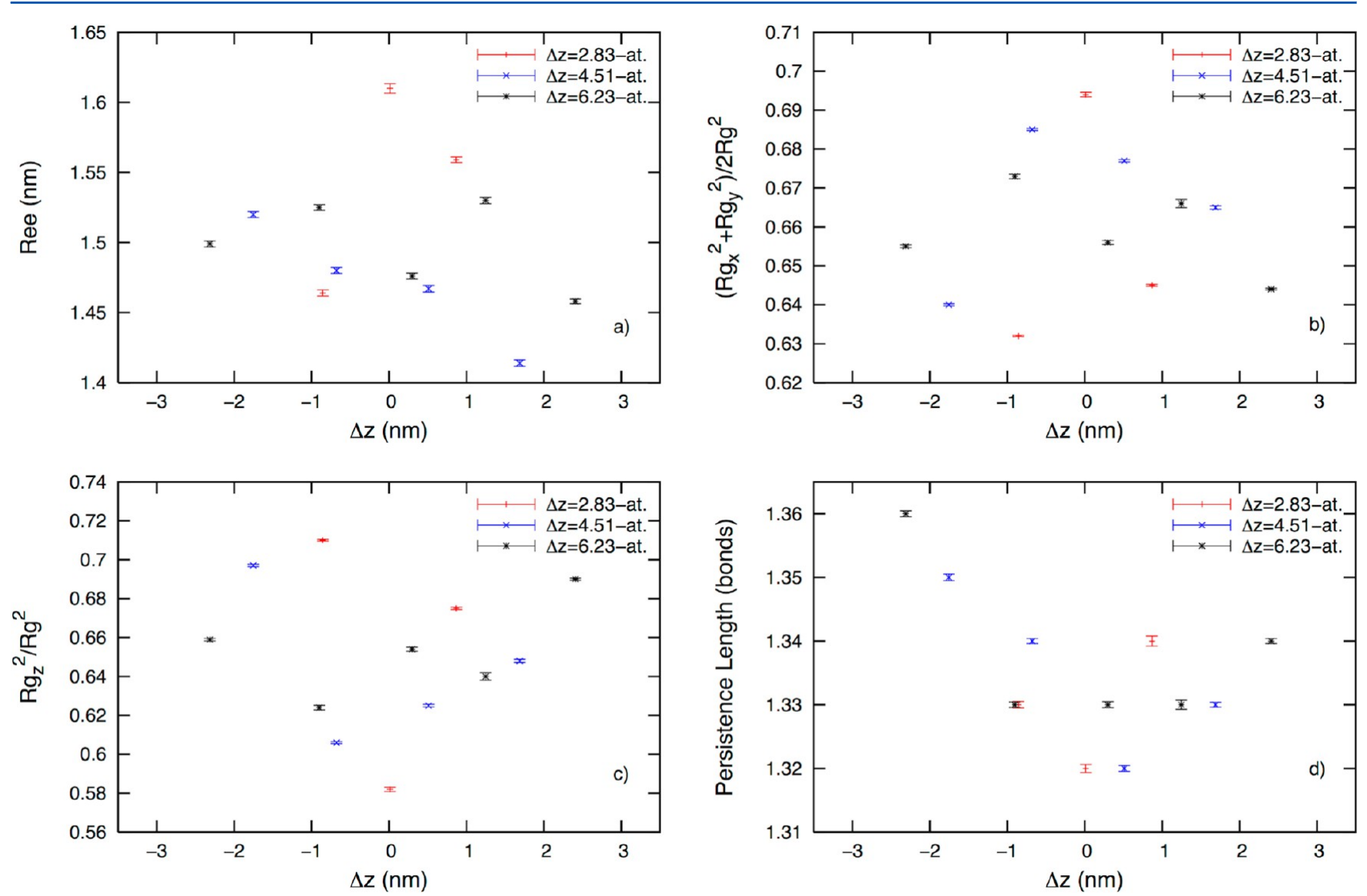

Figure 12. Static properties of the chains as a function of the distance from the walls for different degrees of confinement. (a) End-to-end distance $\left(R_{\mathrm{ee}}\right)$. (b) Normalized parallel component of the radius of gyration. (c) Normalized normal component of the radius of gyration. (d) Persistence length. Standard errors are given as error bars. Centers of the systems are at $z=0$.

surfaces does not change with confinement, while the average dimensions normal to the surfaces are slightly, though not systematically, decreased. These results are in good agreement with the literature for both simulations and experiments. ${ }^{44-47}$ Since the systems studied are not ultraconfined (as the smallest $H / R_{\mathrm{g}_{\mathrm{b}}}$ ratio is 3.9), significant changes in the dimensions of the chains normal to the surfaces would not be expected.

The changes in chain conformations/dimensions with respect to the distance from the surfaces were also investigated. To achieve this, the distance between the walls in $z$-direction was divided into slices of 0.6 to $1.5 \mathrm{~nm}$ in each confined system. For the last $2 \mathrm{~ns}$ of each production run (which is much smaller than the diffusion times of chains that are $\sim 20 \mathrm{~ns}$ for $\Delta z=4.51$ and $\Delta z=6.23$ systems and $\sim 100$ ns for $\Delta z=2.83$ ), chains were indexed into groups according to the slice that their centers of mass were found. The static properties were then calculated for each group. The changes in end-to-end distances $\left(R_{\mathrm{ee}}\right)$, normalized parallel $\left(\left(R_{\mathrm{g} x}{ }^{2}+R_{\mathrm{g} y}{ }^{2}\right) / 2\left(R_{\mathrm{g}}{ }^{2}\right)\right)$ and normal components $\left(R_{\mathrm{gz}}{ }^{2} / R_{\mathrm{g}}{ }^{2}\right)$ of the radii of gyration are given in Figure 12, parts a-c, respectively. It is observed that $R_{\mathrm{ee}}$ and $\left(R_{\mathrm{g} x}{ }^{2}+R_{\mathrm{g} y}{ }^{2}\right) / 2\left(R_{\mathrm{g}}{ }^{2}\right)$ get smaller from the middle of the system toward the surfaces, while $R_{\mathrm{g} z}{ }^{2} / R_{\mathrm{g}}{ }^{2}$ gets larger. The trend is rather pronounced for the smallest confinement system $(\Delta z=$ $2.83)$, while it is less prominent for the largest confinement system $(\Delta z=6.23)$. Evidently, the results are supporting the data in Table 2. We should note that the trend seen in Figure 12 , parts $a-c$, is contrary to some literature results on confined polymer melts, ${ }^{19,20,22}$ which observed that the chains were elongated in the $x-y$ plane, while they were compressed in the normal direction toward the surfaces. However, this was mainly because the chains adopted completely flattened conformations near the surfaces. In contrast, we see that the chains were aligned only weakly parallel to the surfaces even in the smallest confinement system and no transformation from a spherical to an oblate conformation near the walls existed. There may be a number of factors determining the behavior of the polymers close to the surfaces such as the structure and chemistry of the 
walls, its interactions with the polymer, chemistry and size of the polymer, degree of confinement, etc. Possibly the presence of toluenes causes the difference in the orientation of PS chains near the surfaces in our system, compared to that in a confined polymer melt. As, there are more components in a solution than in a melt; there is an adsorption competition to decrease the free energy in the system. Considering dissimilar mobilities of the molecules resulting from the differences in sizes and polymer connectivity of the monomers in polymer chains, the preferred adsorption of toluenes on the surfaces is apprehendable.

Figure $12 \mathrm{~d}$ shows the change in persistence length as a function of distance from the surfaces. We observe that persistence lengths are larger for the chains, which are closer to the surfaces in all systems. As the persistence length is a measure of flexibility, it is not surprising to see such an increase closer to the walls; i.e., chains become less flexible due to the geometrical restrictions near the surfaces.

3.1.3. Dynamic Properties. In order to investigate the effect of confinement on the global relaxation of PS chains, we use end-to-end vector autocorrelation functions (ACFs). Figure 13a shows the ACFs (calculated using second order Legendre polynomials) of PS chains under confinement and in the bulk. The results for the bulk and $\Delta z=4.51$ system have been reported previously. ${ }^{3}$ It is seen that the chains are fully relaxed in all systems as all ACFs decay to zero. Relaxation is the fastest in the bulk, while it slows down with degree of confinement, as the rotational motion is dramatically restricted close to surfaces.
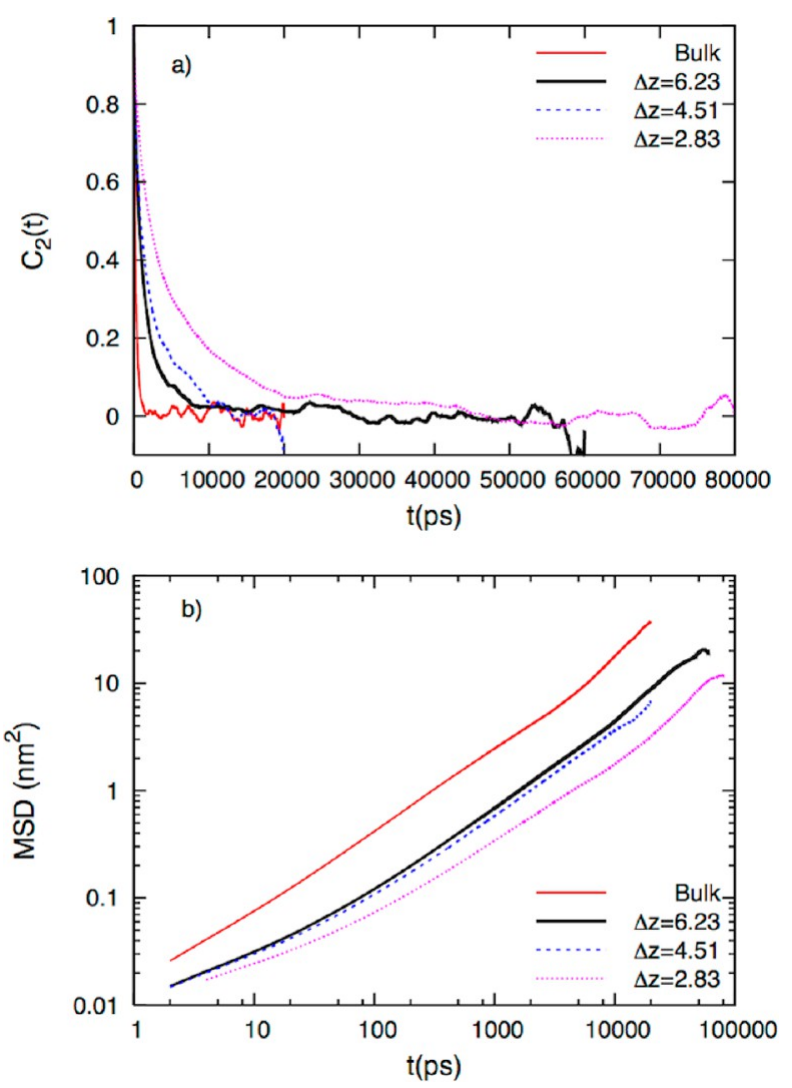

Figure 13. Dynamic properties of PS under different degrees of confinement (and in bulk) in atomistic simulations: (a) end-to-end vector autocorrelation functions $\left(C_{2}(t)\right)$ based on second order Legendre polynomials; (b) mean square displacements (MSDs) of the center of mass of the central five PS monomers.
Since the systems studied here have the same concentration, the number of chains in close contact with the surfaces increases as confinement increases, leading to a decrease in the number of freely rotating/translating chains. Similar effects on the relaxation dynamics of polymer chains have been reported in the literature. ${ }^{19,20,48}$ However, we should note that they studied the effect of pore size while keeping the parallel component of the pressure constant to ensure that the solution is in equilibrium with the bulk. This was not our main concern as the primary objective of this study is to test the applicability of our CG confined concentrated solution model to different confinement levels at constant concentration (and temperature).

The effect of confinement on the translation of PS chains is captured by mean square displacements (MSD) and diffusion coefficients. In order to reduce end effects, MSDs (Figure 13b) were calculated for the central five monomers of a chain. Diffusion coefficients were calculated based on MSDs (Table 3 ). The results indicate similar behavior in the diffusive motion as in the chain relaxation; i.e. diffusion is fastest in the bulk and decreases dramatically with confinement.

Table 3. Diffusion Coefficients of PS Chains as a Function of Confinement

$\begin{array}{cc}\text { system } & D \times 10^{-5}\left(\mathrm{~cm}^{2} / \mathrm{s}\right) \\ \Delta z=2.83 & 0.010 \pm 0.002 \\ \Delta z=4.51^{a} & 0.060 \pm 0.010 \\ \Delta z=6.23 & 0.068 \pm 0.001 \\ \text { bulk }^{a} & 0.329 \pm 0.004\end{array}$

${ }^{a}$ Values were reported previously in ref 3 .

3.2. Coarse-Grained Simulations. Transferability of The Confined Solution Model To Different Confinement Levels. Here we test the transferability of our parent CG confined model on different confinement levels; we have employed the model on systems $\Delta z=2.83$ and $\Delta z=6.23$, of which the results of atomistic simulations are reported above. The details of the initial configuration preparation for the new CG systems and the simulation parameters are explained below.

3.2.1. Simulations Details. The preparation of the initial configuration, choice of the mapping scheme/point, wall superatoms and the simulation parameters for the parent CG confined system were described in detail in a previous publication $^{3}$ and summarized in section 2 together with the technical considerations during the optimization process. Here, we describe how the CG confined systems of smaller and larger confinement levels were generated. We use the mapping scheme described in section 2.2 for atactic polystyrene. CG walls are made of beads of the mass of a PS monomer (104.06 $\mathrm{amu}$ ) on a hexagonal lattice. For the smaller confinement system $(\Delta z=2.83)$, each surface is composed of 2400 beads, while for the larger confinement system $(\Delta z=6.23)$ each surface is made of 1024 beads. In the formation of our CG surfaces, we paid attention to reproduce the corresponding reference systems' wall dimensions as closely as possible. However, due to the limitations imposed by the choice of our CG wall beads, a perfect match with the reference values could not be achieved. Though, they were sufficiently close to the atomistic surfaces (see Table 1). The separation distances between the walls (as defined by the centers of wall beads) were set as 3.21 and $6.53 \mathrm{~nm}$ for the smaller and larger confinement systems, respectively, considering the larger 
effective sizes of CG superatoms and the need to reproduce the effective (true) concentration of the reference system. ${ }^{3}$ Table 1 also shows the effective distances between the CG walls (as determined by the density profiles along the $z$-direction), which the effective (true) concentration calculations of our CG systems were based on.

Initial configurations to the CG simulations were prepared by the extraction of superatom centers from the output configurations of their corresponding reference atomistic simulations for both systems. The parent confined solution system model was used in all simulations. The production runs for both of the systems lasted for $50 \mathrm{~ns}$ and the analyses were made in the last $5 \mathrm{~ns}$ of the whole trajectory.

3.2.2. Reproduction of the Local Structure. Reproduction of the local structure was checked by calculating bonded and nonbonded conformational distributions and density profiles. We start our discussion on the results with density profiles as they assist in discussion of the reproduction of the conformational distributions. Figure 14 shows density profiles obtained in the CG simulations and reference atomistic simulations. For the smallest confinement system, the density of PS monomers near the surfaces is reproduced well while the density in the
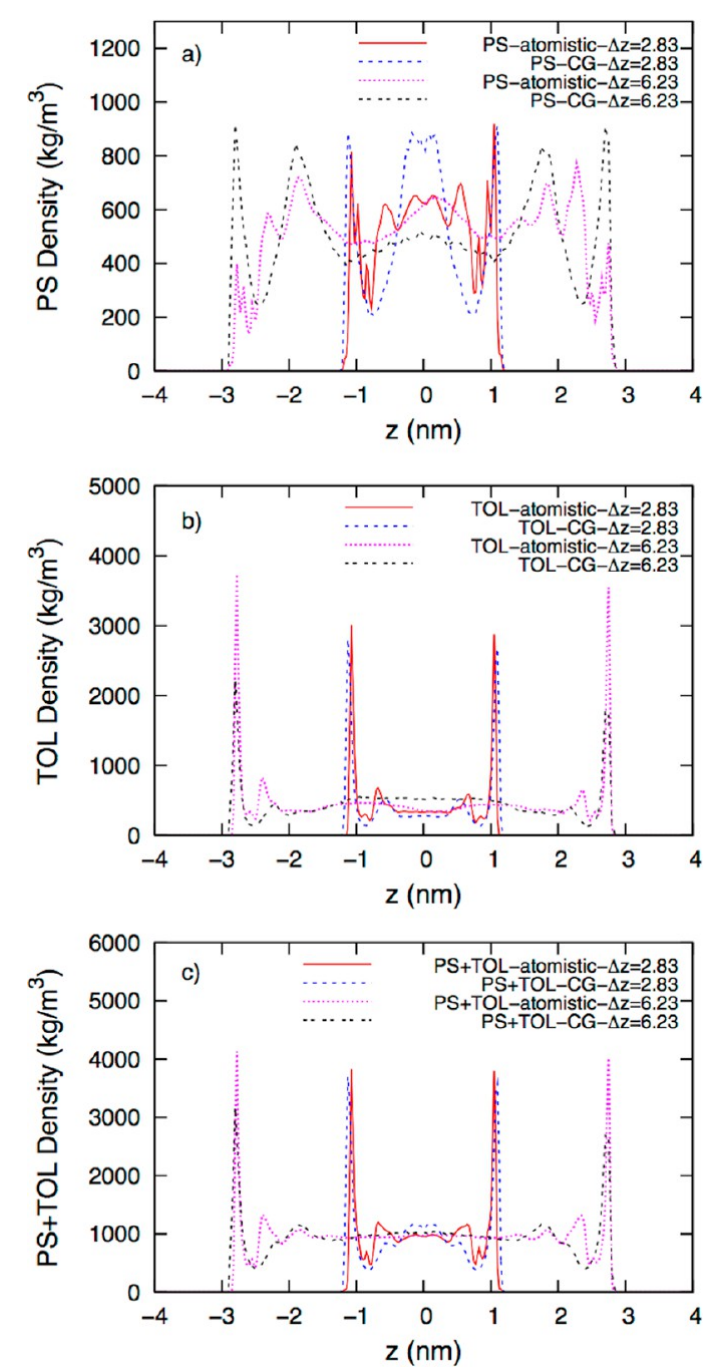

Figure 14. Reference atomistic versus CG: (a) PS, (b) TOL, and (c) PS + TOL density profiles (in direction of the normal to the surfaces) for different degrees of confinement. center of the system is overestimated. There is a slight underestimation of TOL density profile; but reproduction is satisfactory. The density profile of the whole system (Figure 14c) indicates that the layering behavior of the system is produced correctly; however the system has moved a little toward the middle compared to the reference atomistic system. This is obviously due to the localization of PS chains in the center of the system. The reason for that might be the slightly decreased solvation capability of TOL molecules as seen in Figure 16c. As the PS monomers are less solvated by toluenes, the nonbonded interactions between each other become more effective leading to formation of clusters.

In the largest confinement system, the density of PS monomers near the surfaces is overestimated, while the density of PS in the middle of the system is underestimated. Correspondingly, the density of TOL molecules near the walls is underestimated while it is overestimated in the middle. The reason for this behavior becomes clear when we compare the reference (atomistic) PS and TOL density profiles of this system with the parent confined system (see Figure 7). The density of PS near the walls is much lower and the density of TOL molecules near the walls is much larger than in the parent confined system $(\Delta z=4.51)$. We remind that the whole CG force field was optimized based on the structure of the atomistic parent confined system. Therefore, the PS-wall interactions (which are obviously too strong) and TOL-wall interactions (too weak) in CG simulations of the larger confinement system led to overestimation/underestimation of the PS/TOL densities closer to the walls. On the other hand, the estimated densities near the surfaces are good in the smallest confinement system, which is because the surface concentrations of both PS and TOL in the atomistic $\Delta z=2.83$ system are not quantitatively much different than the atomistic parent system (see the labels in Figure 7).

The bonded conformational distributions are shown in Figure 15 with end monomers excluded. It is clear that bond distributions in both of the CG confined systems are reproduced perfectly by the parent CG confined model (Figure $15 a, b)$. In an earlier study, we have shown that bond length between PS monomers is well-defined regardless of the environment that the polymers are in (a melt, dilute and concentrated solution, and a confined concentrated solution). ${ }^{3}$ Also bending angle distributions obtained from the CG simulations are in very good agreement with their corresponding reference structures (Figure 15c,d). Finally, as torsion interactions are the softest bonded interactions this is a more critical test (Figure 15d,e). The deviations from the reference structures are still within $0.6 \%$ as measured by the merit function (eq 6).

Next, we address the capability of our parent CG confined model to reproduce the nonbonded distributions. Parts a, c, and e of Figure 16 show the RDFs of the smallest confinement system $(\Delta z=2.83)$ and the corresponding reference RDFs. The nearest neighbors to a PS monomer (1st two peaks in PSPS RDF) are reproduced very well. However, a slight underestimation of the probability density of farther monomers is observed in the region $\sim 1<r<\sim 1.75 \mathrm{~nm}$. This might be due to the more localized organization of PS chains in the middle of the system compared to the reference system as shown by the density profile in Figure 14a. As the chains cluster in the center, deeper and wider wells are formed in the density profile, which points to a deficiency of PS monomers/chains in that region. Therefore, we can say that there are less distant 

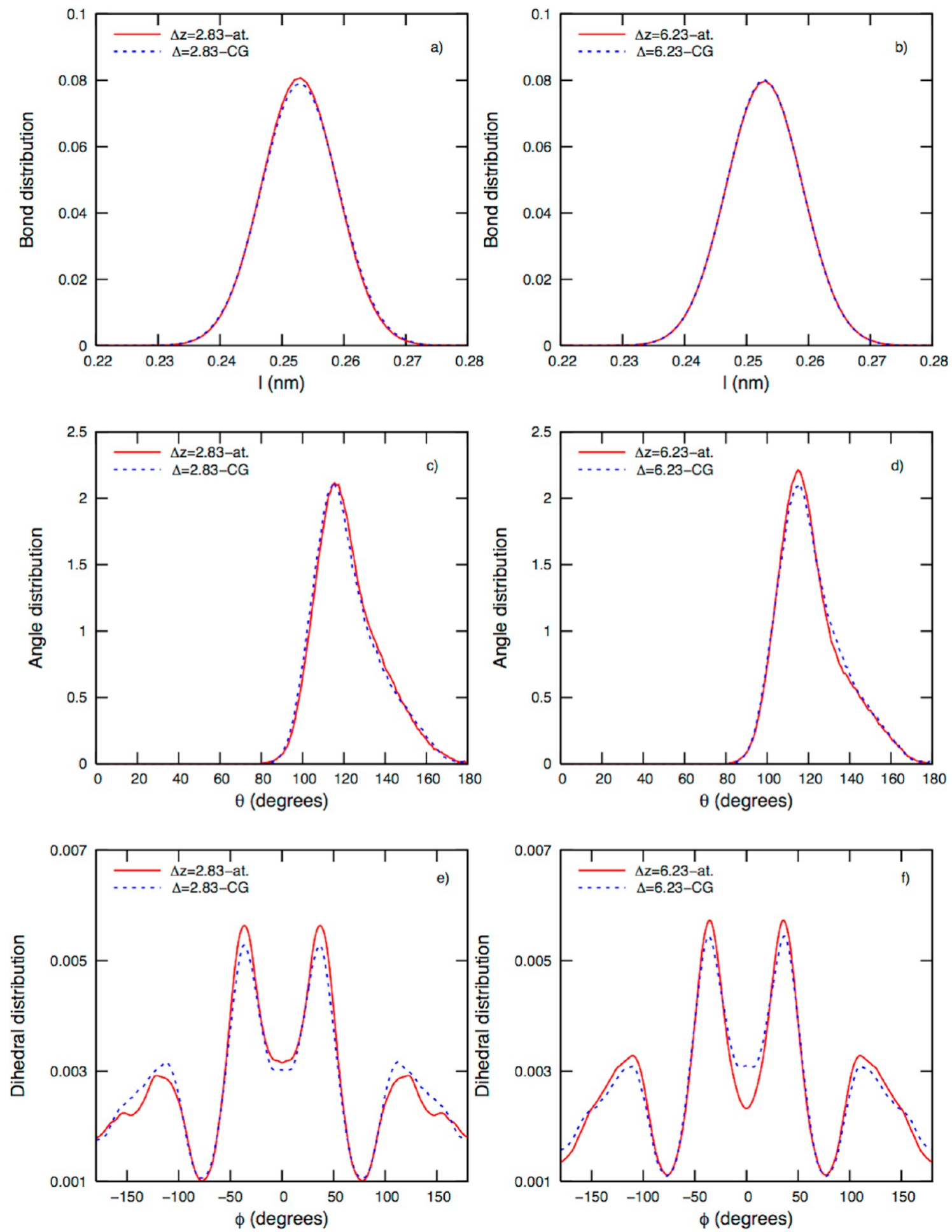

Figure 15. Reproduction of the reference (atomistic) bonded conformational distributions of the smallest $(\Delta z=2.83)$ and the largest confinement $(\Delta z=6.23)$ systems by the parent CG confined model. Key: (a, b) bond length; (c, d) bending angle; (e, f) dihedral angle probability distributions.

neighbors to a monomer in the CG system than in the reference, leading to the discrepancy in PS-PS RDFs. Less significant deviations from the references are observed in PSTOL and TOL-TOL RDFs. Figure 16c suggests that solvent quality of TOL is slightly decreased in the CG system, due to the more localized organizations of PS chains in the middle; i.e. toluenes do not have the same degree of freedom to approach PS monomers as in the reference atomistic system.
For the PS-PS RDF in the largest confinement system $(\Delta z$ $=6.23$ ), we observe that the reproduction of local structures is very good (except the minor discrepancy in the first peak, which is probably due to assigning spherically symmetric CG potentials to PS). The discrepancy at larger distances is greater than in the smallest confinement system. The reason for this larger underestimation in the probability density of the farther monomers may be the overestimated PS densities near the walls and underestimated densities in the middle of the system 

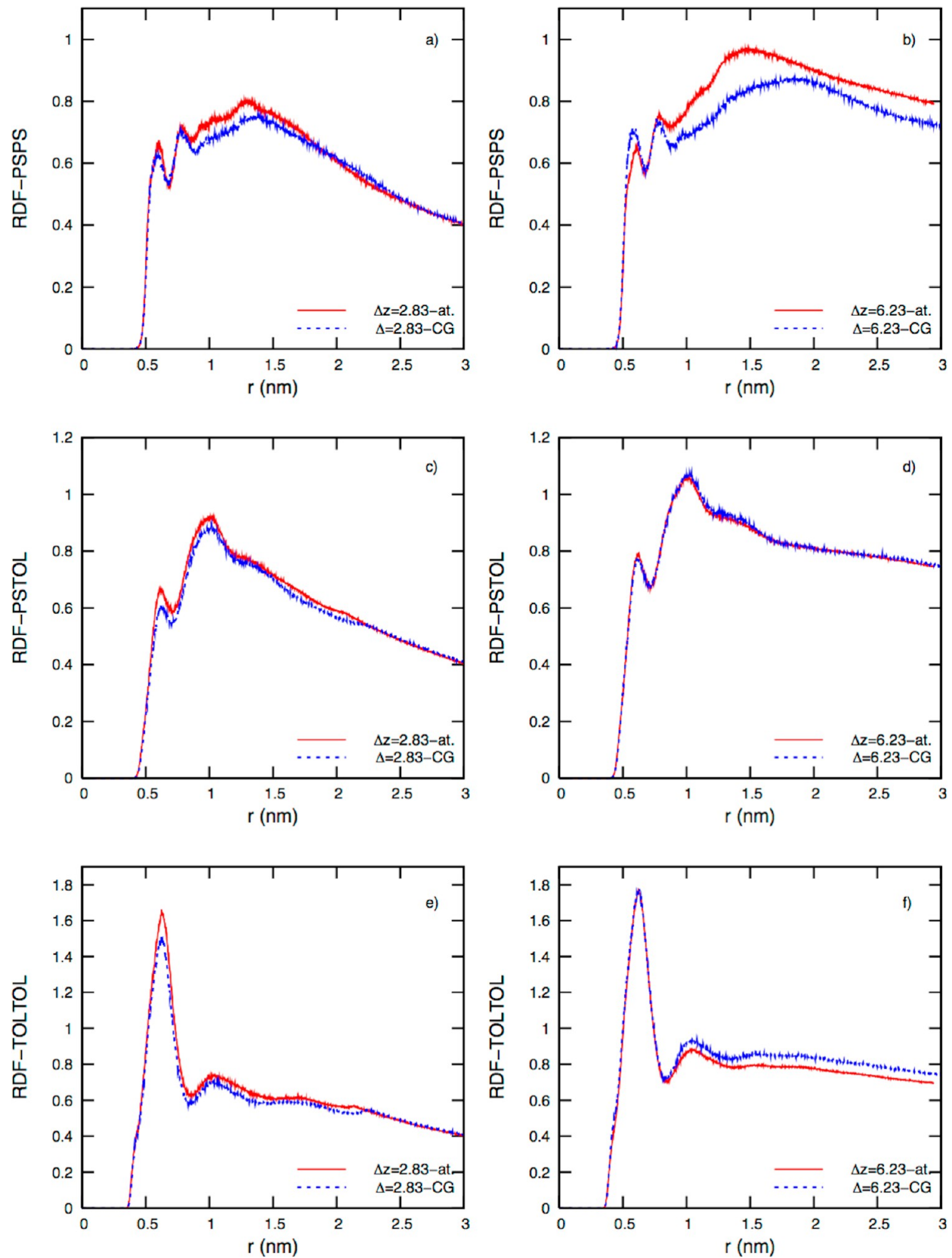

Figure 16. Reproduction of the reference (atomistic) nonbonded conformational distributions of the smallest $(\Delta z=2.83)$ and the largest confinement $(\Delta z=6.23)$ systems by the parent CG confined model. Key: (a, b) PS-PS; (c, d) PS-TOL; (e, f) TOL-TOL radial distribution functions.

(Figure 14a). Similar to the case in the smallest confinement system, there are much less distant neighbors to the monomers in the CG system than in the reference system. This effect is more significant in the largest confinement system, because overestimation of the PS densities near walls is significantly greater compared to that in the CG $\Delta z=2.83$ system. We observe that the reproduction of PS-TOL RDF is excellent. This might be due to a balance between the effect of increase in the density of PS monomers near the walls (and the deficiency in the middle) and the effect of increase in the density of TOL 
molecules in the middle (and the deficiency near the walls). Finally, we observe a slight overestimation in the packing of toluenes around themselves at larger distances $(r>1 \mathrm{~nm})$ (Figure 16f).

In summary, we have observed that the reproduction of the local structure in the atomistic $\Delta z=2.83$ system by our parent CG confined model works fairly well. We believe this is because density profiles/surface concentrations in both of the atomistic systems $(\Delta z=2.82$ and $\Delta z=4.51)$ are similar. In contrast, reproduction of the local structure in the atomistic $\Delta z=6.23$ system was rather poor. Although the conformational distributions do not deviate more than $0.9 \%$ from the references, the density profiles show significant differences. This is because of the similar argument that surface concentrations and degree of alignment of the chains near the surfaces in two of the reference systems $(\Delta z=6.23$ and $\Delta z$ $=4.51$ ) are different from each other. Therefore, the interaction potentials (between the walls and the monomers/molecules) optimized based on the structure on one system may be too strong or not strong enough to capture the reference structure of the other system, even at the same concentration.

3.2.3. Reproduction of the Global Structure. In section 3.1.2, the static properties of PS chains were reported based on all-atom data. For a CG system all static properties are calculated based on superatom centers. Our mapping scheme assigns the Carbon atoms at which the phenyl rings are attached as superatom centers. Therefore, calculations of endto-end distances are based on these last carbon atoms at both ends and will be shorter, since the actual atoms are further apart from the superatom centers. ${ }^{3}$ In order to compare the atomistic with CG data, we recalculated the static properties of PS chains in the confined atomistic systems based on CG mapping points (Table 4 "scaled atomistic data").

Table 4. Reference (Atomistic) Coarser-Scaled Static Properties of the PS Chains versus Values Obtained with the Parent CG Confined Model for Each Confinement Level

\begin{tabular}{|c|c|c|c|}
\hline system & $\begin{array}{l}R_{\mathrm{g}}(\mathrm{nm}) \text { (scaled } \\
\text { atomistic and CG } \\
\text { data) }\end{array}$ & $\begin{array}{l}R_{\mathrm{ee}}(\mathrm{nm})(\mathrm{scaled} \\
\text { atomistic and CG } \\
\text { data) }\end{array}$ & $\begin{array}{c}R_{\mathrm{ee}}{ }^{2} / R_{\mathrm{g}}{ }^{2} \text { (scaled } \\
\text { atomistic and CG } \\
\text { data) }\end{array}$ \\
\hline $\begin{array}{l}\Delta z=2.83 \\
\quad \text {-atomistic }\end{array}$ & $0.59 \pm 0.005$ & $1.491 \pm 0.026$ & 6.386 \\
\hline $\begin{array}{l}\Delta z=2.83 \\
-\mathrm{CG}\end{array}$ & $0.627 \pm 0.009$ & $1.656 \pm 0.049$ & 6.976 \\
\hline $\begin{array}{r}\Delta z=4.51 \\
\text {-atomistic }\end{array}$ & $0.583 \pm 0.005$ & $1.457 \pm 0.039$ & 6.245 \\
\hline $\begin{array}{l}\Delta z=4.51 \\
-\mathrm{CG}\end{array}$ & $0.618 \pm 0.008$ & $1.625 \pm 0.047$ & 6.914 \\
\hline $\begin{array}{l}\Delta z=6.23 \\
\quad \text {-atomistic }\end{array}$ & $0.584 \pm 0.006$ & $1.436 \pm 0.045$ & 6.046 \\
\hline $\begin{array}{l}\Delta z=6.23 \\
-\mathrm{CG}\end{array}$ & $0.615 \pm 0.009$ & $1.618 \pm 0.048$ & 6.922 \\
\hline
\end{tabular}

Comparison of the coarser-scaled values from the atomistic simulations with the calculated values from CG simulations show that the global structure of PS are slightly overestimated for all CG systems, which is probably due to the increased end effects with coarse-graining. As PS chains used in this study are oligomers (composed of 15 monomers), it is expected that end monomers show some excluded volume effects. This effect will be larger in a CG system, because the end monomers (superatoms) are larger as they are treated as perfect spheres throughout the simulations. ${ }^{3}$ Considering the same degree of overestimation in the parent confined model (in comparison to its atomistic values), we can say that the estimation of static properties for the CG $\Delta z=2.83$ and $\Delta z=6.23$ systems are reasonable.

We should note that the $R_{\mathrm{ee}}{ }^{2} / R_{\mathrm{g}}{ }^{2}$ data in the last column of Table 4 do not represent the correct conformation of the chains as they are calculated based on the mapping points. They indicate that the chains are in extended conformations (because the ratio is greater than 6) although they are in more compact form in reality (based on all-atom data, see column 4 of Table 2 ). Therefore, one should be careful not to make conclusions about the conformations of polymer chains based solely on CG data but should also seek for the atomic scale results, especially for oligomers.

\section{CONCLUSIONS}

Recently, we developed a coarse-grained (CG) model for a concentrated polystyrene (PS) solution under confinement using the iterative Boltzmann inversion (IBI). ${ }^{3}$ Reproductions of the local and global structure of the polymers as well as the efficiency of the model in terms of dynamics were discussed in detail. In this study, we establish the limits of the technique in the coarse-graining of confined polymeric systems. The aim is 2-fold: to get a better insight about the limits of the method by going deeper into the technical considerations and challenges faced in the course of the optimization process, and to investigate the transferability of the CG model developed on that specific system (referred as the "parent CG confined model") on similar systems at constant concentration and temperature, but at different degrees of confinement.

In the first part of the manuscript, we refer to some technical points and difficulties encountered in the implementation of the method on our system. Among those, the choice of the initial guess potentials is an important one. We observed that with the use of Boltzmann inverse functions of the target conformational distributions from the atomistic simulations as the initial guess potentials, which is the common practice in the implementation of IBI, the system turned into a semiglassy state (polymers were crystallized in the solution). Alternatively, the system was still a solution when a different set of initial guess potentials was used; i.e. previously optimized CG effective potentials of the same system without confinement. ${ }^{3}$ Clearly, this was due to the incorrect dihedral potential and imbalance between the initial guess potentials used for PS-PS, PS-TOL, and TOL-TOL interactions in the former implementation, as the potentials of mean force (Boltzmann inverse functions) account neither for the higher order nonpairwise correlations nor the PS orientation correlations in the system. Therefore, we suggest that, for coarse-graining a confined polymeric system with IBI, it might be a good idea to coarse-grain the same system without confinement first. One can use the optimized potentials as the initial guess potentials for the iterations on the confined system then since there is no strong theoretical basis in IBI for the use of Boltzmann inverse functions as the initial potentials. ${ }^{12}$ Apart from this, we also discuss how the cross-correlations differ for only the surface segments compared to the overall and the order of optimization. Typically, the order of optimization is stretching bond, bending angle, nonbonded and dihedral angle potentials based on the strength of the interactions. However, experience showed that, if the initial dihedral distribution is substantially different from the target distribution, it would be beneficial to change the order of the last two terms for the first few iterations, as this might be crucial to correct the radial 
distribution function (RDF) between PS monomers in local distances.

The test of transferability of the parent CG confined model to different degrees of confinement (at constant concentration and temperature) is the other goal of the study. Specifically, we are interested to see if a CG force field developed for a confined system by a purely structure-based coarse-graining technique, such as iterative Boltzmann inversion (IBI), is sensitive to changes in the degree of localization or arrangement of polymers near the surfaces although the concentration is kept constant. For this purpose, we have performed reference atomistic simulations on systems of two different confinement levels; i.e., systems with a smaller and a larger confinement level (compared to the parent reference confined system). The differences in the structure and dynamics of the polymers in comparison to the parent reference confined system and an unconfined (bulk) system of same concentration are also addressed.

Local structures of the polymers were characterized through bond/angle/dihedral angle distributions, radial distribution functions (RDFs) and density profiles, while the global structures were described in terms of end-to-end distances $\left(R_{\mathrm{ee}}\right)$ and radii of gyration $\left(R_{\mathrm{g}}\right)$. Orientation of chains with respect to the surface normal was captured by the calculations of order parameter for end-to-end vectors of the polymers. Density profiles indicated that the degree of localization of PS and toluene (TOL) molecules near the surfaces were significantly different in the largest confinement system than in the rest. This was due to the relatively (but still weakly) more normal orientation of PS chains along the surfaces, which led to a preferential localization of TOL molecules near the surfaces. Analysis of the static properties indicated that the chains were compact (compared to a Gaussian chain) in all systems including the bulk. However, they showed more elongated conformations as the degree of confinement increased. This does not mean that the chain dimensions parallel to the surfaces were increased as no significant changes in average chain dimensions either parallel or normal to the surfaces were observed. For a closer look on the effect of confinement on chain dimensions, the changes in chain conformations/dimensions with respect to the distance from the surfaces were also investigated. It was observed that the end-to-end distance and the parallel component of the radius of gyration slightly decreased while the normal component of the radius of gyration slightly increased from the middle of the system toward the surfaces with the trend being less prominent as the degree of confinement decreases. The results are contrary to literature data on confined polymer melts, where the chains adopted fully parallel (flattened) orientations in close to the surfaces. ${ }^{19,20,22}$ Though, our order parameter results showed that the PS chains were aligned only weakly parallel to the surfaces even in the smallest confinement system. We suppose this is a consequence of the preferred localization of TOL molecules in the vicinity of the surfaces resulting from the adsorption competition to decrease the total free energy. Therefore, we believe this study is an important contribution to the understanding of the differences in polymer conformations/ orientations close to surfaces in a concentrated solution from that in a melt. To the best of our knowledge, there is no computational study that explores this difference. In addition to the static properties, rotational and translational dynamics of PS chains were examined through end-to-end vector autocorrelation functions and mean-square displacements. It was observed that relaxation of end-to-end vectors and diffusion of the chains were drastically slowed down by the presence of walls.

The transferability of our parent CG confined model to different confinement levels (keeping the concentration and temperature constant) was tested by employing the parent force field in CG simulations of the above-mentioned systems. Results showed that reproducibility of the local structure in the smallest confinement system was fairly well. We believe this is because the degree of arrangement of PS chains and TOL molecules close to the surfaces in the reference atomistic smaller confinement system is similar to that of the parent confined atomistic system (as indicated by the density profiles). In contrast, reproduction of the local structure in the largest confinement system by our parent CG confined model was poor, which is because both PS and TOL density profiles were different from those of the parent reference confined system. Therefore, interaction potentials between the walls and the monomers/TOL molecules, which were optimized based on the structure of the parent system, failed to mimic the real interactions between the walls and the rest of the larger confinement system. In conclusion, our results show that the degree of arrangement of monomers/molecules near the surfaces is an important factor affecting the transferability of a CG force field, which is developed by a structure-based coarsegraining technique such as IBI, to different degrees of confinement.

\section{ASSOCIATED CONTENT}

\section{S Supporting Information}

Comparison of the overall joint conformational probability distributions (of PS monomers/superatoms) in the atomistic simulations and in coarse-grained simulations. This material is available free of charge via the Internet at http://pubs.acs.org.

\section{AUTHOR INFORMATION}

\section{Corresponding Author}

*E-mail: (R.F.) rfaller@ucdavis.edu.

\section{Notes}

The authors declare no competing financial interest.

\section{ACKNOWLEDGMENTS}

We would like to gratefully acknowledge Dr. David M. Huang and Dr. Petra Träskelin for providing scripts used for data analysis and Dr. Halil Mecit Oztop for his support in the statistical analysis of the data. This work was supported by the U.S. Department of Energy, Office of Science, Basic Energy Sciences (grant number DE-FG02-06ER46340). BB was also partially supported by The Scientific and Technological Research Council of Turkey (TUBITAK).

\section{REFERENCES}

(1) Bayramoglu, B.; Faller, R. Phys. Chem. Chem. Phys. 2011, 13 (40), 18107-18114.

(2) Advincula, R. C.; Brittain, W. J.; Caster, K. C.; Ruhe, J., Polymer Brushes. In Wiley-VCH: Weinheim, Germany, 2004.

(3) Bayramoglu, B.; Faller, R. Macromolecules 2012, 45, 9205-9219.

(4) Faller, R. Polymer 2004, 45 (11), 3869-3876.

(5) Theodorou, D. N. Chem. Eng. Sci. 2007, 62 (21), 5697-5714.

(6) Sun, Q.; Faller, R. Macromolecules 2006, 39 (2), 812-820.

(7) Megariotis, G.; Vyrkou, A.; Leygue, A.; Theodorou, D. N. Ind. Eng. Chem. Res. 2011, 50 (2), 546-556.

(8) Milano, G.; Goudeau, S.; Muller-Plathe, F. J. Polym. Sci., Part B: Polym. Phys. 2005, 43 (8), 871-885. 
(9) Soper, A. K. Chem. Phys. 1996, 202, 295.

(10) Reith, D.; Putz, M.; Muller-Plathe, F. J. Comput. Chem. 2003, 24

(13), 1624-1636.

(11) Henderson, R. L. Phys. Lett. A 1974, 49, 197-198.

(12) Chan, E. R.; Striolo, A.; McCabe, C.; Cummings, P. T.; Glotzer,

S. C. J. Chem. Phys. 2007, 127 (11).

(13) Ghosh, J.; Faller, R. Mol. Simul. 2007, 33 (9-10), 759-767.

(14) Sun, Q.; Faller, R. Comput. Chem. Eng. 2005, 29 (11-12), $2380-2385$.

(15) Sun, Q.; Ghosh, J.; Faller, R. In Coarse-Graining of Condensed Phase and Biomolecular Systems; Voth, G. A., Ed.; CRC Press: Boca Raton, FL, 2009; pp 69-82

(16) Ghosh, J.; Wong, B. Y.; Sun, Q.; Pon, F. R.; Faller, R. Mol. Simul. 2006, 32 (3-4), 175-184.

(17) Qian, H.-J.; Carbone, P.; Chen, X.; Karimi-Varzaneh, H. A.; Liew, C. C.; Muller-Plathe, F. Macromolecules 2008, 41 (24), 99199929.

(18) Carbone, P.; Varzaneh, H. A. K.; Xiaoyu, C.; Muller-Plathe, F. J. Chem. Phys. 2008, 128 (6).

(19) Eslami, H.; Karimi-Varzaneh, H. A.; Muller-Plathe, F. Macromolecules 2011, 44 (8), 3117-3128.

(20) Aoyagi, T.; Takimoto, J.; Doi, M. J. Chem. Phys. 2001, 115 (1), $552-559$.

(21) Varnik, F.; Binder, K. Int. J. Mater. Res. 2009, 100 (11), 14941502 .

(22) Varnik, F.; Baschnagel, J.; Binder, K. Eur. Phys. J. E 2002, 8 (2), 175-192.

(23) Eslami, H.; Karimi-Varzaneh, H. A.; Muller-Plathe, F. Macromolecules 2011, 44 (8), 3117-3128.

(24) Baschnagel, J.; Varnik, F. J. Phys.: Condens. Matter 2005, 17 (32), R851-R953.

(25) Sun, Q.; Faller, R. J. Phys. Chem. B 2005, 109 (33), 1571415723.

(26) Hess, B.; Bekker, H.; Berendsen, H. J. C.; Fraaije, J. J. Comput. Chem. 1997, 18 (12), 1463-1472.

(27) Schuttelkopf, A. W.; van Aalten, D. M. F. Acta Crystallogr., Sect. D: Biol. Crystallogr. 2004, 60, 1355-1363.

(28) Traskelin, P.; Kuhl, T. L.; Faller, R. Phys. Chem. Chem. Phys. 2009, 11 (47), 11324-11332.

(29) Darden, T.; York, D.; Pedersen, L. J. Chem. Phys. 1993, 98 (12), 10089-10092.

(30) Essmann, U.; Perera, L.; Berkowitz, M. L.; Darden, T.; Lee, H.; Pedersen, L. G. J. Chem. Phys. 1995, 103 (19), 8577-8593.

(31) Berendsen, H. J. C.; Postma, J. P. M.; Vangunsteren, W. F.; Dinola, A.; Haak, J. R. J. Chem. Phys. 1984, 81 (8), 3684-3690.

(32) Nose, S. Mol. Phys. 1984, 52 (2), 255-268.

(33) Hoover, W. G. Phys. Rev. A 1985, 31 (3), 1695-1697.

(34) Hess, B.; Kutzner, C.; van der Spoel, D.; Lindahl, E. J. Chem. Theory Comput. 2008, 4 (3), 435-447.

(35) Van der Spoel, D.; Lindahl, E.; Hess, B.; Groenhof, G.; Mark, A. E.; Berendsen, H. J. C. J. Comput. Chem. 2005, 26 (16), 1701-1718. (36) Lindahl, E.; Hess, B.; van der Spoel, D. J. Mol. Model. 2001, 7 (8), 306-317.

(37) Theodorou,D. N. In Computer Simulations in Condensed Matter Systems: From Materials to Chemical Biology; Springer: Berlin, 2006.

(38) Peter, C.; Kremer, K. Soft Matter 2009, 5 (22), 4357-4366.

(39) Sun, Q.; Faller, R. J. Chem. Theory Comput. 2006, 2 (3), 607-

615.

(40) Faller, R.; Schmitz, H.; Biermann, O.; Muller-Plathe, F. J. Comput. Chem. 1999, 20 (10), 1009-1017.

(41) Harmandaris, V. A.; Adhikari, N. P.; van der Vegt, N. F. A.; Kremer, K. Macromolecules 2006, 39 (19), 6708-6719.

(42) Binder, K.; Paul, W.; Virnau, P.; Yelash, L.; Muller, M.; Gonzalez MacDowell, L. In Coarse-Graining of Condensed Phase and Biomolecular Systems; Voth, G. A., Ed.; CRC Press: Boca Raton, FL, 2009; pp 399414.

(43) Doi, M.; Edwards, S. F., The Theory of Polymer Dynamics; Clarendon Press: Oxford, U.K., 1986.
(44) Jones, R. L.; Kumar, S. K.; Ho, D. L.; Briber, R. M.; Russell, T. P. Nature 1999, 400 (6740), 146-149.

(45) Jones, R. L.; Kumar, S. K.; Ho, D. L.; Briber, R. M.; Russell, T. P. Macromolecules 2001, 34 (3), 559-567.

(46) Winkler, R. G.; Gerstmair, A.; Reineker, P.; Matsuda, T.; Yoon, D. Y. Int. J. Quantum Chem. 1994, 52 (2), 437-456.

(47) Pakula, T. J. Chem. Phys. 1991, 95 (6), 4685-4690.

(48) Eslami, H.; Muller-Plathe, F. J. Phys. Chem. B 2009, 113 (16), $5568-5581$. 